\title{
Munc13-Independent Vesicle Priming at Mouse Photoreceptor Ribbon Synapses
}

\author{
Benjamin Cooper, ${ }^{1}$ Maike Hemmerlein, ${ }^{2}$ Josef Ammermüller, ${ }^{3}$ Cordelia Imig, ${ }^{1}$ Kerstin Reim, ${ }^{1}$ Noa Lipstein, ${ }^{1}$ \\ Stefan Kalla, ${ }^{1}$ Hiroshi Kawabe, ${ }^{1}$ Nils Brose, ${ }^{1,4}$ Johann Helmut Brandstätter, ${ }^{2}$ and Frédérique Varoqueaux ${ }^{1,4}$ \\ ${ }^{1}$ Department of Molecular Neurobiology, Max Planck Institute of Experimental Medicine, D-37075 Göttingen, Germany, ${ }^{2}$ Animal Physiology, Department \\ of Biology, University of Erlangen-Nuremberg, D-91058 Erlangen, Germany, ${ }^{3}$ AG Neurobiology, University of Oldenburg, D-26111 Oldenburg, Germany, \\ and ${ }^{4}$ Deutsche Forschungsgemeinschaft Research Center for Molecular Physiology of the Brain, D-37073 Göttingen, Germany
}

Munc13 proteins are essential regulators of exocytosis. In hippocampal glutamatergic neurons, the genetic deletion of Munc13s results in the complete loss of primed synaptic vesicles (SVs) in direct contact with the presynaptic active zone membrane, and in a total block of neurotransmitter release. Similarly drastic consequences of Munc13 loss are detectable in hippocampal and striatal GABAergic neurons. We show here that, in the adult mouse retina, the two Munc13-2 splice variants bMunc13-2 and ubMunc13-2 are selectively localized to conventional and ribbon synapses, respectively, and that ubMunc13-2 is the only Munc13 isoform in mature photoreceptor ribbon synapses. Strikingly, the genetic deletion of ubMunc13-2 has little effect on synaptic signaling by photoreceptor ribbon synapses and does not prevent membrane attachment of synaptic vesicles at the photoreceptor ribbon synaptic site. Thus, photoreceptor ribbon synapses and conventional synapses differ fundamentally with regard to their dependence on SV priming proteins of the Munc13 family. Their function is only moderately affected by Munc13 loss, which leads to slight perturbations of signal integration in the retina.

\section{Introduction}

Vesicular neurotransmitter release at presynaptic active zones is mediated by SNARE complexes, whose activity is tightly regulated by multiple proteins to provide the speed, endurance, and accuracy required for efficient excitation-secretion coupling (Wojcik and Brose, 2007; Verhage and Sørensen, 2008; Gundelfinger and Fejtova, 2011; Südhof and Rizo, 2011). Among these, members of the Munc13 protein family are essential for synaptic vesicle (SV) membrane attachment and priming: in their absence, central synapses do not contain any release-competent SVs and transmitter release is completely blocked (Varoqueaux et al., 2002; Siksou et al., 2009).

The mammalian genome contains three closely related Munc13 genes, Munc13-1, Munc13-2, and Munc13-3 (Brose et al., 1995). The corresponding gene products share a common do-

Received Aug. 17, 2011; revised March 15, 2012; accepted April 3, 2012.

Author contributions: B.C., K.R., N.B., J.H.B., and F.V. designed research; B.C., M.H., J.A., C.I., K.R., N.L., S.K., and F.V. performed research;S.K., H.K., N.B., and J.H.B. contributed unpublished reagents/analytic tools; B.C., M.H., J.A., C.I., K.R., N.L., S.K., N.B., J.H.B., and F.V. analyzed data; B.C., N.B., J.H.B., and F.V. wrote the paper.

This work was funded by the Max Planck Society (N.B.), the Cure Autism Now Foundation (F.V.), Deutsche Forschungsgemeinschaft (DFG) Center for Molecular Physiology of the Brain (F.V., N.B.), and DFG Grant BR 1643/4-1. We are grateful to Dietmar Riedel (Göttingen, Germany) and Stephan Eimer (Göttingen, Germany) for expert advice and access to their instruments, and to Katrin Willig and Stefan Hell (Göttingen, Germany) for access to and support with their STED microscope. We thank the staff from the sequencing facility, and Thea Hellmann and Klaus Hellmann for excellent technical help.

Correspondence should be addressed to either of the following Johann Helmut Brandstätter, Department of Biology, Animal Physiology, University of Erlangen-Nuremberg, Staudtstrasse 5, D-91058 Erlangen, Germany. E-mail: jbrandst@biologie.uni-erlangen.de; or Frédérique Varoqueaux, Max Planck Institute of Experimental Medicine, Department of Molecular Neurobiology, Hermann-Rein-Strasse 3, D-37075 Göttingen, Germany. E-mail: varoqueaux@em.mpg.de.

S. Kalla's present address QIAGEN GmbH, OIAGEN Strasse 1, D-40724 Hilden, Germany.

DOI:10.1523/JNEUROSCI.4240-11.2012

Copyright $\odot 2012$ the authors $\quad 0270-6474 / 12 / 328040-13 \$ 15.00 / 0$ main structure in their C-terminal halves, which is functionally essential for priming activity (Basu et al., 2005; Stevens et al., 2005) and exhibits structural homology to vesicle tethering factors (Li et al., 2011). Two more distantly related genes encode the Munc13-like proteins Munc13-4 and Bap3/Baiap3 (Koch et al., 2000), which play key roles in non-neuronal cells (e.g., in the immune system) (Feldmann et al., 2003; Pivot-Pajot et al., 2008).

The Munc13-1 and Munc13-3 genes each encode one major protein product, while the Munc13-2 gene produces two major splice variants with different N-terminal extensions, the brainspecific bMunc13-2 (Brose et al., 1995; Augustin et al., 1999a; Betz et al., 2001) and the ubiquitously expressed ubMunc13-2 (Song et al., 1998; Betz et al., 2001). The most extensively studied isoform, Munc13-1, is widely expressed in the brain (Augustin et al., 1999b) and specifically localized to active zones (Betz et al., 1998; Rhee et al., 2002). In addition to conserved C terminals, Munc13-1 and ubMunc13-2 also share a highly conserved $\mathrm{N}$-terminal region (Betz et al., 1998) that contains a C2 domain (C2A), mediates binding to RIM (Rab3-interacting molecule) (Andrews-Zwilling et al., 2006; Wang et al., 2009), CAST (CAZassociated structural protein), Bassoon, and Piccolo (Wang et al., 2009), and a calmodulin-binding domain with a regulatory function (Dimova et al., 2006). To some extent, Munc13-1 and ubMunc13-2 can functionally replace each other, and the two isoforms have often been studied indifferently (Wang et al., 2009; Shin et al., 2010). However, experiments in cultured neurons showed that Munc13-1 and ubMunc13-2 confer differential short-term plasticity characteristics to the synapses they equip (Rosenmund et al., 2002). Despite such striking functional differences, little is known about the distribution of Munc13-1 and ubMunc13-2 in vivo. 
The adult mouse retina, with well defined populations of structurally and functionally distinct conventional and ribbon chemical synapses, is ideally suited to study the distribution and function of Munc13 isoforms. Conventional chemical synapses of amacrine cells are specialized for phasic transmitter release, photoreceptor and bipolar cell ribbon synapses for graded and sustained release (tom Dieck and Brandstätter, 2006; Matthews and Fuchs, 2010). We investigated Munc13 isoforms in the adult mouse retina with a special focus on the two Munc13-2 splice variants, bMunc13-2 and ubMunc13-2. Our findings indicate that exocytosis at photoreceptor ribbon synapses differs from exocytosis at conventional synapses with respect to their requirement for Munc13 family priming proteins.

\section{Materials and Methods}

Retinal tissue preparation. Adult mice (8-12 weeks of age) were killed, and the eyes were quickly removed. Eyecups were immersion-fixed in $4 \%$ paraformaldehyde (supplemented with $0.1 \%$ glutaraldehyde for postembedding immunogold labeling) in $0.1 \mathrm{M}$ phosphate buffer (PB) for 20 min. After PB wash, the retinas were dissected out, immersed until saturation in graded sucrose (for light microscopy) or glycerol (for electron microscopy) solutions ( $10-30 \%, \mathrm{w} / \mathrm{v})$, and frozen.

Antibodies. Munc13 isoform-specific antibodies were generated in rabbit against FPLC-purified N-terminal protein fragments as described previously (Varoqueaux et al., 2005). An additional bMunc13-2-specific antibody was raised in guinea pig using recombinant GST-bMunc13-2 (residues 1-305). Primary antibodies were used at the following dilutions: polyclonal rabbit anti-Munc13-1 [batch " 40 " or " 41 "; Western blot (WB), 1:2000; immunohistochemistry (IHC), 1:6000], antiubMunc13-2 (batch "52"; WB, 1:1000; IHC, 1:6000), anti-bMunc13-2 (batch "50"; WB, 1:1000; IHC, 1:6000), anti-Munc13-3 (batch "48"; WB, 1:1000; IHC, 1:6000); guinea pig anti-bMunc13-2 (IHC, 1:1000), antiVGluT1 (IHC, 1:4000; Millipore Bioscience Research Reagents); monoclonal mouse anti-pan-Munc13 (WB, 1:200; BD Biosciences Transduction Laboratories), anti-CtBP2 (IHC, 1:12,000; BD Biosciences), and antibassoon (IHC, 1:2000; Enzo Life Sciences).

Western blotting analysis. Retina, brain, and olfactory bulb homogenates were prepared from mice of the indicated genotypes. Primary hippocampal neurons of the indicated genotypes were prepared from E18 mice according to standard procedures (Rosenmund et al., 2002) and cultured on poly-D-lysine-coated coverslips at a density of 312.5 cells/ $\mathrm{mm}^{2}$ for $9 \mathrm{~d}$ at $5 \% \mathrm{CO}_{2}$. After washing in PBS, neurons were harvested and homogenized in a buffer containing $50 \mathrm{~mm}$ Tris, $150 \mathrm{~mm} \mathrm{NaCl}, 2 \mathrm{~mm}$ EDTA, and 1\% SDS, pH 7.4. Munc13 expression patterns were investigated using standard SDS-PAGE, Western blotting, and immunoblotlabeling procedures, with antibody dilutions as indicated above.

Immunohistochemistry and light-microscopic analysis. Fourteenmicrometer-thick vertical cryostat sections of the retina were incubated overnight at $4^{\circ} \mathrm{C}$, in primary antibody mix in $\mathrm{PB}$ containing 3\% normal goat serum and $0.1 \%$ Triton X-100. Incubation in secondary antibodies coupled to Alexa 488 or Alexa 555 (1:2000; Invitrogen), Cy5 (1:2000; Millipore Bioscience Research Reagents), or Atto647N (1:100; kindly provided by S. Hell, Max Planck Institute for Biophysical Chemistry, Göttingen, Germany) was then performed for $1 \mathrm{~h}$ at room temperature. Labeled sections were examined with a confocal laser-scanning microscope (LSM5; Zeiss; or TCS SP5 STED; Leica; all confocal images shown are single-plane images, in which the same volume of the sections was imaged for each channel; see Figs. 1, 2, 5, 6) or a Zeiss Axio Imager Z1 coupled to an Apotome (Zeiss; see Fig. 4). Image contrast and brightness were adjusted with Photoshop, and figures were compiled with Illustrator (CS2; Adobe).

RNA and cDNA preparation from mouse retina, and cloning of ubMunc13-2 transcripts. To prepare RNA, two 5-month-old Munc13-2 knock-out (KO) mice (Varoqueaux et al., 2002) were killed, and the retinas were removed and flash frozen in liquid nitrogen. One milliliter of TRIzol reagent (Invitrogen) was added to the frozen retinas, followed by homogenization for $30 \mathrm{~s}$ using an Ultra-Turrax (IKA). One milliliter of
Table 1. Primers for $P C R$ amplification of ubMunc13-2 transcripts

\begin{tabular}{lll}
\hline Exon & $5^{\prime}-3^{\prime}$ sequence & Direction \\
\hline $9(1)$ & GGCAGTTCCCATGACTCTTG & Forward \\
$9(2)^{a}$ & CCTTGATTATCCAGAGCGTC & Forward \\
$10(1)$ & GGTATGGCTCCTCCTGTAATG & Forward \\
$10(2)^{a}$ & CCAGTATCATGAGCAAGATG & Forward \\
27 & GGAGCCACTTCACCTTGAAG & Reverse \\
31 & GGACCCTCAGTTGCTGCATG & Reverse \\
$32^{a}$ & CCAGAACTGTATTCAGTTTCACC & Reverse \\
33 & GGTCGCAGCACACTATCTG & Reverse \\
$34^{a}$ & CCATGGTGTTCATCACCAC & Reverse \\
35 & GGCAGCAGTTAAGATCAGCTG & Reverse \\
36 & GGACAGCACACTGCTTGG & Reverse \\
40 & GGTCACTTCCACAAAAGGC & Reverse \\
41 & GGAGAATGGTCATGCCTG & Reverse \\
\hline
\end{tabular}

${ }^{a}$ Reverse oligos for exons 32 and 34 were combined with forward oligos 9 (2) and 10 (2).

homogenate was then mixed with $200 \mu$ of chloroform and incubated for $3 \mathrm{~min}$ at room temperature. To separate the phases, samples were centrifuged at $12,000 \times g$ for $15 \mathrm{~min}$ at $4^{\circ} \mathrm{C}$. The aqueous phases were placed into new tubes. The same volume of $70 \%$ ethanol was then added to each sample, and the whole content of the tube was transferred to an RNeasy column (QIAGEN). RNA purification was then continued according to the manufacturer's instructions. The concentration of purified RNA was measured photometrically. For first-strand cDNA synthesis, the following components were added to nuclease-free microcentrifuge tubes: 50 pmol of oligo-d $\mathrm{T}_{16,18,20}$ primer or $136 \mathrm{ng}$ of random primers; $350 \mathrm{ng}$ of total RNA; $2 \mu \mathrm{l}$ of $10 \mathrm{~mm} \mathrm{dNTP}$ mix, and sterile DEPC-treated water to $13 \mu \mathrm{l}$. Mixtures were heated to $65^{\circ} \mathrm{C}$ for $5 \mathrm{~min}$ and then incubated on ice for at least $1 \mathrm{~min}$. The content of the tubes was collected by brief centrifugation and the following components were added: $4 \mu \mathrm{l}$ of $5 \times$ First-Strand Buffer, $1 \mu \mathrm{l}$ of $0.1 \mathrm{~m}$ DTT, $1 \mu \mathrm{l}$ of RNaseOUT Recombinant RNase Inhibitor (Invitrogen), and $1 \mu \mathrm{l}$ of SuperScript III RT (200 U/ $\mu \mathrm{l})$. The tube containing the random primers was then incubated at $25^{\circ} \mathrm{C}$ for $5 \mathrm{~min}$. All tubes were subsequently incubated at $50^{\circ} \mathrm{C}$ for $60 \mathrm{~min}$. The reaction was inactivated by heating at $70^{\circ} \mathrm{C}$ for 15 min. RNA was removed by RNaseH treatment at $37^{\circ} \mathrm{C}$ for $20 \mathrm{~min}$. ubMunc13-2-specific primers (Table 1) were selected on the basis of the published mouse cDNA sequence (GenBank accession no. NM_021468.2). Amplifications were done using MG078 Velocity polymerase (Bioline). All PCR products were cloned into the $\mathrm{PCR}-\mathrm{XL}-\mathrm{TOPO}$ vector (Invitrogen); clones containing inserts were purified using the PureLink Quick Plasmid Miniprep Kit (Invitrogen) and sequenced.

Electron-microscopic analysis of ubMunc13-2 distribution. Fixed, cryoprotected retinas were plunge-frozen in liquid propane, processed by freeze-substitution (AFS; Leica), and embedded in Lowicryl HM20 (Electron Microscopy Sciences) as described previously (Matsubara et al., 1996). Labeling was performed on ultrathin sections (70 nm) incubated $2 \mathrm{~h}$ at room temperature in primary antibody (anti-ubM13-2; batch " 52 "; 1:2000) in Tris-buffered saline containing 0.05\% Triton $\mathrm{X}-100$ (TBST) and $0.1 \%$ BSAc (Aurion), and $1 \mathrm{~h}$ in $10 \mathrm{~nm}$ gold-coupled secondary goat anti-rabbit IgG (1:20; Aurion) in TBST containing 1\% BSAc and $0.05 \%(\mathrm{w} / \mathrm{v})$ polyethyleneglycol. Sections were contrasted with uranyl acetate and lead citrate and observed in a LEO 912AB transmission electron microscope (Zeiss). Digital pictures were taken with a ProScan CCD camera coupled to the Esivision software (Olympus).

Ultrastructural analysis of synaptic vesicle docking in high-pressurefrozen photoreceptor terminals. Samples from three pairs of Munc13-2 KO and wild-type littermates (8-12 weeks of age) were prepared for highpressure freezing as described previously (Siksou et al., 2009; Möbius et al., 2010). Briefly, fresh pieces of retina were dissected out and frozen under high pressure (HPM 010; Baltec), followed by freeze-substitution and Epon embedding. Electron-tomographic analysis of docked vesicles was restricted to vertically oriented rod ribbon synapses exhibiting excellent ultrastructural preservation and a visible cytomatrix at the active zone (CAZ)/arciform density. For qualitative analysis, 150-nm-thick sections were coated on both surfaces with 10-nm-diameter protein A-gold particles (Aurion) to serve as fiducial markers, and photoreceptor termi- 
nals were imaged in a Philips CM120 BioTwin transmission electron microscope (Philips) operating at $120 \mathrm{kV}$. Single-axis tilt series $(-65$ to $+65^{\circ}$ ) were acquired at $27,500 \times$ magnification with a TemCam F224A camera (TVIPS). Final tomographic volumes reconstructed for qualitative analysis had an isotropic voxel size of $1.3 \mathrm{~nm}$. To obtain larger tomographic volumes for statistical analysis, fiducial-coated 200-nm-thick sections were imaged in a JEM-2100 transmission electron microscope operating at $200 \mathrm{kV}$. Single-axis tilt series $\left(-60\right.$ to $\left.+60^{\circ}\right)$ were recorded at $25,000 \times$ magnification with an Orius SC1000 camera (Gatan). Statistical analyses were performed on tomographic volumes reconstructed with an isotropic voxel size of $1.6 \mathrm{~nm}$. All tomographic volumes were reconstructed with the IMOD package (Kremer et al., 1996; Mastronarde, 1997) (http://bio3d.colorado.edu/).

Within each tomogram, the region of interest was restricted to the membrane invagination formed at the ribbon site. Three populations of SVs were quantified as follows: (1) the mean number of docked SVs normalized to the length of the sampled presynaptic membrane forming the invagination; (2) the mean number of SVs docked exclusively at the base of the ribbon in direct apposition to the CAZ; and (3) the mean number of undocked SVs possessing physical tethers to the synaptic ribbon normalized to ribbon height. Only SVs observed to be in physical contact with the presynaptic membrane at their midline were considered docked. Tomographic volumes were exported as TIFF image stacks into ImageJ for the quantification of presynaptic membrane and ribbon dimensions. The presynaptic membrane length forming the invagination and the vertical aspect of the synaptic ribbon extending from the CAZ into the cytoplasm were measured every $40 \mathrm{~nm}$ through the $z$-dimension of the tomographic volume and averaged to produce a mean presynaptic membrane length and a mean ribbon height, respectively. The statistical significance of differences in vesicle docking in Munc13-2 KO and wildtype ribbon synapses was determined by two-tailed, unpaired Student's $t$ test.

Electroretinographic recordings. Electroretinographic (ERG) recordings were performed on five pairs of age-matched (8- to 12-week-old) male wild-type or Munc13-2 KO mice that were dark-adapted overnight before commencement of the measurements. Both scotopic and photopic responses were recorded and analyzed as previously described (Reim et al., 2009). Shortly, mice were anesthetized by intraperitoneal injections of xylazine $(50 \mathrm{mg} / \mathrm{kg})$ and ketamine $(20 \mathrm{mg} / \mathrm{kg})$. Pupils were dilated with $1 \%$ atropine sulfate. A gold coil electrode was placed on the cornea, which was moistened with Methocel, and a platinum reference needle electrode was inserted into the skin covering the skull. A platinum grounding needle electrode was inserted into the tail. Mice were placed onto a temperature-regulated platform (Roland Consult). Surgery and subsequent handling were done under dim red darkroom light. Electrical potentials were recorded and bandpass filtered $(1-1000 \mathrm{~Hz})$ using a ML132 Bioamplifier (ADInstruments) connected to a PowerLab system (ADInstruments) and PC. A calibrated Ganzfeld ERG setup was used for light stimulation (Q450; Roland Consult). Ten responses were averaged per intensity. A-wave response amplitudes were measured relative to baseline. The b-wave amplitude was determined from the most negative a-wave trough to the b-wave peak, and b-wave implicit times were measured from stimulus onset to b-wave maximum. At highest intensities, b-wave implicit times could not be measured reliably, due to the superimposed oscillatory potentials, and were therefore not included in the analysis. For analysis of oscillatory potentials (OPs), ERG traces were bandpass filtered $(30-300 \mathrm{~Hz})$. From this filtered response, peak-to-peak amplitudes of OPs were measured. In addition, a time slice from the maximum of the a-wave to $200 \mathrm{~ms}$ was chosen for fast Fourier transformation (FFT) (Reim et al., 2009). In the FFT power spectrum, peak power and frequency at which peak power occurred were measured and plotted against light intensity. Analysis was performed with CHART, version 5 (ADInstruments). Statistical analysis was done with JMP 5.0 (SPSS), and results were plotted using Deltagraph, version 5 (SPSS).

Generation of Munc13-2-EYFP and Munc13-3-EGFP knock-in mice. The targeting vectors for the generation of Munc13-2-EYFP and Munc13-3-EGFP knock-ins were designed following the strategy used for the Munc13-1-EYFP line (Kalla et al., 2006). They were constructed on the basis of two genomic clones in pBluescript (pM13-2-3' and
pM13-3-3') that had been isolated from a $\lambda$ FIXII genomic library of the SV129 mouse strain (Stratagene). In the targeting vector, the STOP codon in the last coding exon of the genes was replaced in-frame by an EYFP/EGFP cDNA fragment from pEYFP/EGFP-N1 (Clontech). By using conventional cloning strategies, a neomycin resistance cassette flanked by two $\operatorname{lox} P$ sites was inserted in sense orientation $3^{\prime}$ of the EYFP/EGFP STOP codon, and two copies of the HSV thymidine kinase gene were attached at the $5^{\prime}$ end of the targeting vector (see Fig. $4 A, E$ ). Mice carrying the mutated Munc13-2-EYFP-Neo or Munc13-3-EGFPNeo genes $\left(+/ \mathrm{m}_{\mathrm{n}}\right)$ were generated by homologous recombination in embryonic stem cells (SV129/ola) and identified by Southern blotting (see Fig. $4 B, F$ ) or PCR. To eliminate deleterious effects of the neomycin resistance gene, heterozygous mice carrying the mutated genes $\left(+/ \mathrm{m}_{\mathrm{n}}\right)$ were crossed with EIIa-cre mice (Lakso et al., 1996). Successfully recombined Munc13-2-EYFP or Munc13-3-EGFP genes in offspring from these interbreedings were genotyped by Southern blotting or PCR. Homozygous mice $(\mathrm{m} / \mathrm{m})$ were used for all experiments.

\section{Results}

\section{Munc13 isoforms are differentially localized in the retina}

In mammals, the four closely related Munc13s Munc13-1, bMunc13-2, ubMunc13-2, and Munc13-3 are involved in exocytosis at central and peripheral synapses (Augustin et al., 1999b, 2001; Varoqueaux et al., 2002, 2005). In the present study, their distribution in the retina was investigated using antibodies raised against protein sequences in their divergent $\mathrm{N}$-terminal region (Fig. 1A) (Varoqueaux et al., 2005). Serum specificity was assessed by comparing data obtained in wild-type versus Munc13-1, Munc13-2, and Munc13-3 single KO mice (Augustin et al., 1999b, 2001; Varoqueaux et al., 2002) (Fig. $1 B-F$ ). The perinatally lethal phenotype of the Munc13-1 deletion-mutant mouse did not permit the collection of retina sections and forced us to use newborn brain homogenate (Fig. $1 B$ ) as the negative control reference sample. Homogenates (Fig. 1C) and sections (Fig. $1 F$ ) from adult retinas were used otherwise. Each serum specifically recognized the Munc isoform/variant it was raised against, on Western blots as well as on sections (Fig. $1 B-F$ ). In addition, the anti-bMunc13-2 antiserum yielded labeling of cell bodies in the inner nuclear layer (INL) and ganglion cell layer (GCL) that was present in the wild-type but also the Munc13-2 $\mathrm{KO}$ retina and thereby considered as nonspecific (Fig. $1 F$ ).

We found that all Munc13 isoforms and major splice variants are abundantly expressed throughout the retina (Fig. $2 A$ ). In the inner plexiform layer (IPL), where bipolar, amacrine, and ganglion cells interact, each Munc13 isoform exhibited a very distinct labeling pattern. Small Munc13-1-immunoreactive clusters were visible throughout the IPL, with lower abundance in the transition zone between the OFF and ON bipolar cell terminal fields (Fig. 2A). Munc13-3-labeled puncta were distributed throughout the IPL, and large immunoreactive clusters were preferentially located in the innermost sublamina of the IPL, indicating a partial association of Munc13-3 with larger bipolar cell terminals (Fig. 2A). The two Munc13-2 splice variants, bMunc13-2 and ubMunc13-2, exhibited a strictly partitioned distribution (Fig. 2A-E). bMunc13-2 immunoreactivity consisted of small synaptic-like puncta organized in four horizontal strata reminiscent of the distribution of inhibitory synapses in the IPL. In contrast to all other isoforms, ubMunc13-2 labeling was present at both synaptic layers of the retina; while immunoreactive puncta were rather uniformly distributed throughout the IPL, they were also present in the outer plexiform layer (OPL), where glutamatergic, ribbon-bearing photoreceptor terminals contact bipolar cell dendrites and horizontal cell processes (Fig. 2A). These distinct staining patterns indicate that the Munc13 isoforms and 
A

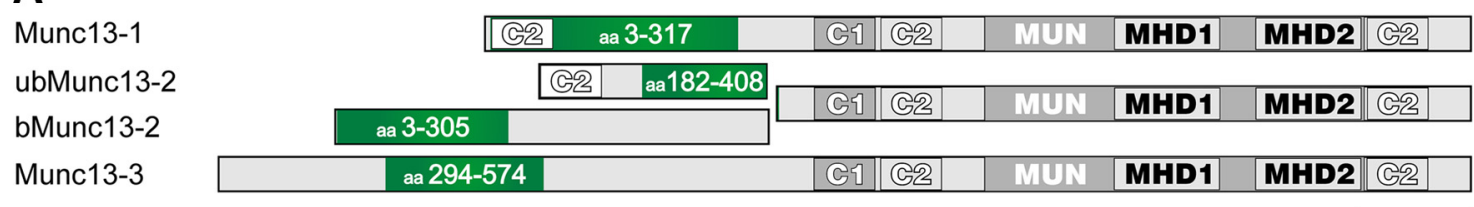

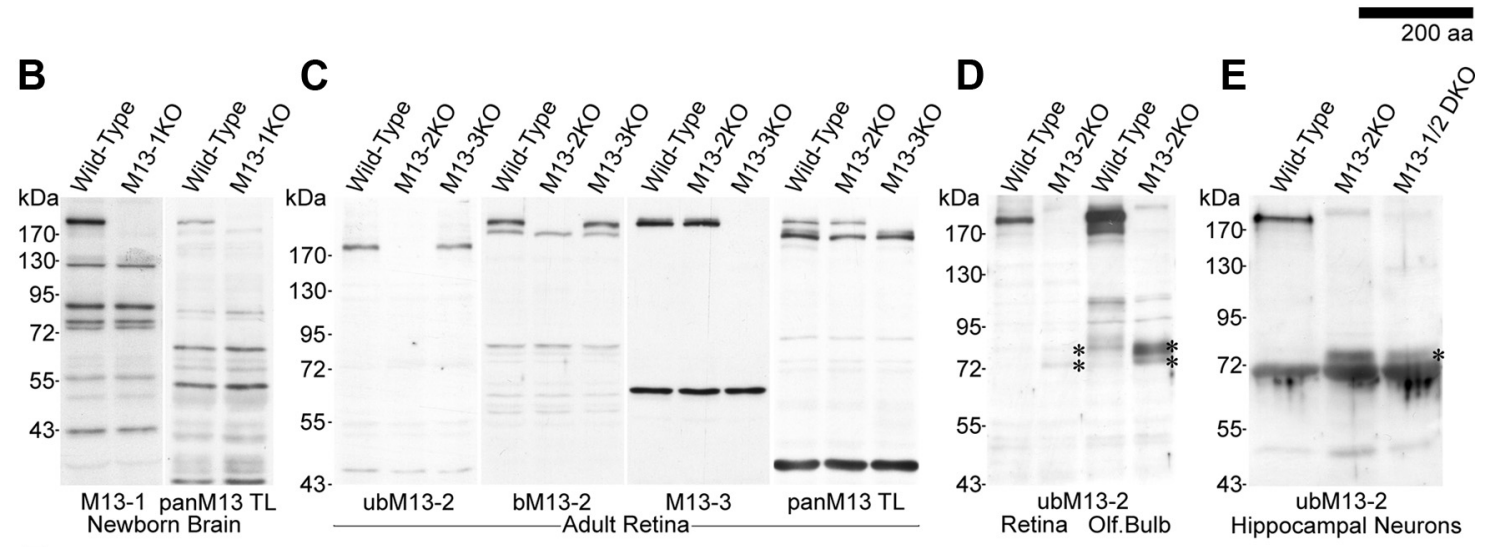

$\mathbf{F}$
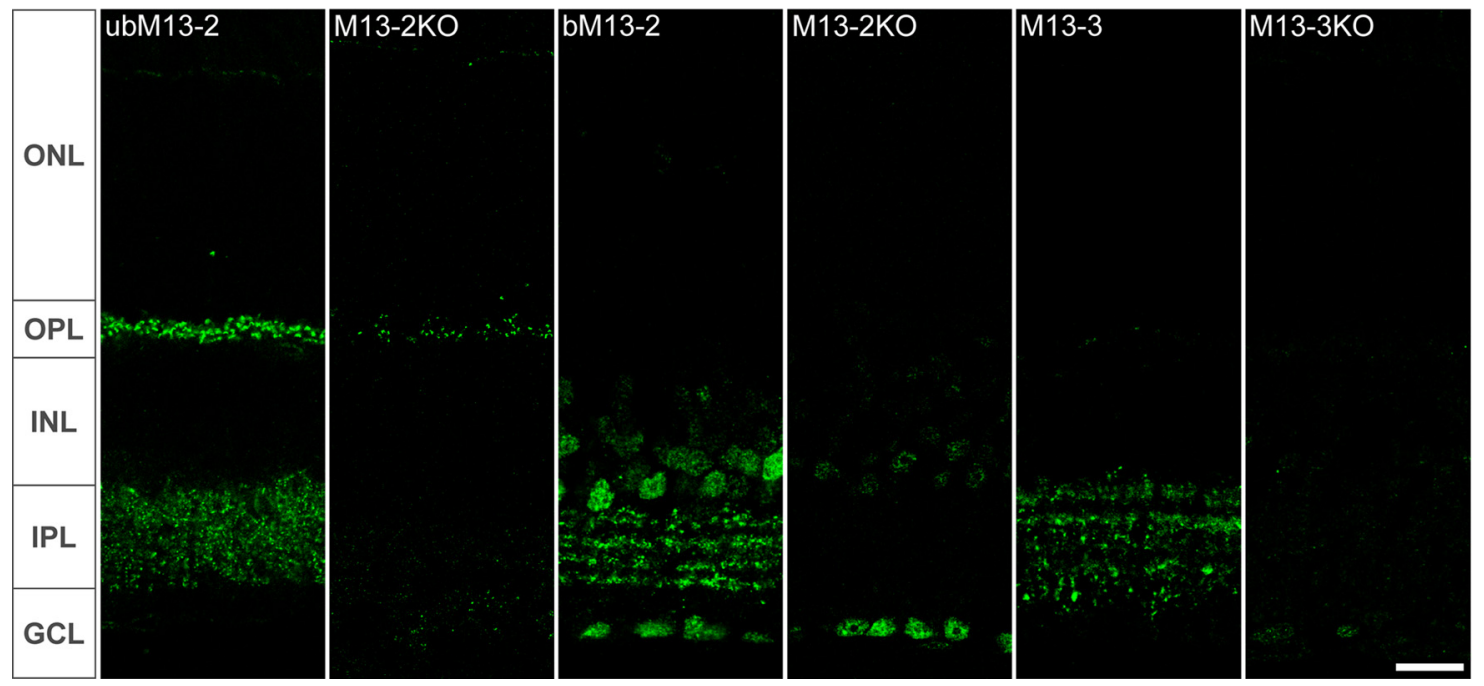

Figure 1. Specificity of Munc13 isoform-specific antisera. $A$, Schematic representation of protein fragments (framed in green) used to raise specific antibodies against the canonical Munc13 isoforms/splice variants. C1, C1 domain; C2, C2 domain; MHD, Munc13 homology domain (Koch et al., 2000); MUN, Mun domain (Li et al., 2011). B-D, Western blot detection of Munc13-1, ubMunc13-2, bMunc13-2, and Munc13-3 using our own and commercial (panMunc13 TL; BD Biosciences) antibodies in homogenates from newborn brain $(\boldsymbol{B})$, and adult retina ( $(\boldsymbol{D}, \boldsymbol{D})$ or olfactory bulb (D) homogenates of the indicated genotypes. The specificity of each antibody is indicated by the absence of corresponding immunoreactive band in extracts from the respective deletion-mutant mice. $\boldsymbol{D}$, Immunoblot labeling with an anti-ubMunc13-2 serum revealed that ubMunc13-2 is strongly expressed in the olfactory bulb and that an unidentified protein is selectively expressed in Munc13-2-deficient but not in wild-type mice (right). A similar fragment was observed in Munc13-2-deficient retina extracts (left). $\boldsymbol{E}$, In homogenates from Munc13-2 K0 and Munc13-1/2 DKO hippocampal neurons, the ubMunc13-2 antibody recognizes a similar fragment. $\boldsymbol{D}, \boldsymbol{E}$, The asterisks indicate the $70-75 \mathrm{kDa}$ fragments recognized by the ubMunc13-2 antibody in samples from Munc13-2-deficient mice. $\boldsymbol{F}$, Single-plane confocal images taken upon immunocytochemical detection of Munc13 isoforms in vertical sections of adult mouse retina of the indicated genotypes. A weak, residual signal was observed for ubMunc13-2 at both the OPL and IPL of the Munc13-2 deletion-mutant retina. This may be consistent with the presence of a protein fragment observed in the Munc13-2 KO by Western blot (D). In contrast, the punctate labelings observed with the antisera directed against bMunc13-2 and Munc13-3 were no longer present in the corresponding K0 retina. Note the occurrence of a nonspecific nuclear labeling obtained upon application of the antiserum directed against bMunc13-2. ONL, INL, Outer and inner nuclear layers; OPL, IPL, outer and inner plexiform layers; GCL, ganglion cell layer. Scale bars, $20 \mu \mathrm{m}$.

splice variants may differentially equip conventional and ribbon synapses in the mammalian retina.

ubMunc13-2 immunostaining unexpectedly yielded a residual, punctate, synaptic-like staining in the OPL and IPL of the Munc13-2 KO (Fig. $1 F$, second panel). Upon long exposure and densitometric analysis, Western blot detection of ubMunc13-2 uncovered the presence a $70-75 \mathrm{kDa}$ protein product in the Munc13-2 KO that is not detectable in wild-type mouse and is expressed at $3.6 \%$ of the wild-type ubMunc13-2 level (Fig. 1D, left lanes). It is likely that this protein is responsible for the resid- ual staining observed in sections (Fig. $1 F$ ). In homogenates from olfactory bulbs, where ubMunc13-2 is most abundantly expressed, a similar protein fragment (Fig. $1 D$, right lanes) is found at $7.5-8.0 \%$ of the wild-type level, as quantified using fluorescently labeled secondary antibodies.

Since we cannot exclude that it may correspond to a truncated protein resulting from the targeting strategy used to generate the Munc13-2 KO, we next generated cDNA from RNA samples of Munc13-2 KO retinas and PCR-amplified candidate ubMunc13-2 fragments (see Materials and Methods) (Table 1, 


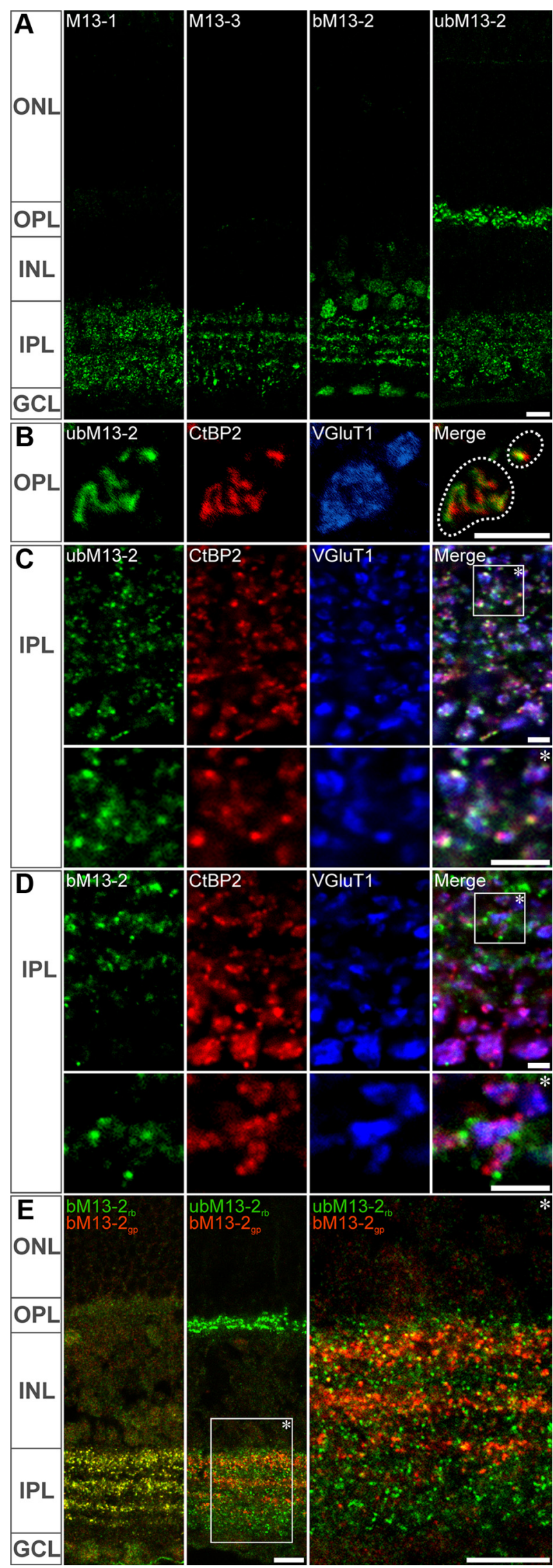

Figure 2. Munc13 isoforms are differentially localized in the mouse retina. Single-plane confocal images of Munc13 labelings. A, Munc13-1, ub- and bMunc13-2, and Munc13-3 exhibited distinct punctate labeling patterns at the plexiform layers of the retina suggestive
Fig. 3A). We found evidence for the presence of seven different ubMunc13-2 transcripts in the Munc13-2 KO retina (Fig. 3B). Yet none of them encodes a protein of this size-nor of higher molecular weight (Fig. 1D). Hence, the identity of the fragment detected remains unclear, and it might alternately represent the upregulated expression of a homologous, yet unrelated protein. Importantly, however, a residual N-terminal ubMunc13-2 fragment of this size would not be expected to have priming activity (Basu et al., 2005; Stevens et al., 2005) (see Discussion) or rescue the function of the protein, as reflected by the total block of SV fusion in hippocampal neurons of the Munc13-1/2 double KO (Varoqueaux et al., 2002), in which a similar residual product was also observed (Fig. 1E). Moreover, this fragment appears also in Munc13-2 KO hippocampal neurons (Fig. 1E), and studies of Munc13-2 KO mice do not report any dominant-negative effect. The Munc13-2 KO mouse therefore remains a valid model for the study of bMunc13-2 and ubMunc13-2 loss of function.

We subsequently focused on the differential distribution of the two Munc13-2 splice variants ubMunc13-2 and bMunc13-2 (Fig. $2 B-E$ ). In triple labeling experiments, we investigated their association with the ribbon marker CtBP2 (Schmitz et al., 2000) and the marker for glutamatergic photoreceptor and bipolar cell terminals VGluT1 (Johnson et al., 2003) at synapses of the OPL (Fig. 2B) and the IPL (Fig. 2C). ubMunc13-2 codistributed with CtBP2 and VGluT1 in both plexiform layers (Fig. 2B,C), while bMunc13-2 did not (Fig. 2D). The strict partitioning of ubMunc13-2 and bMunc13-2 distribution in the IPL was further validated upon colabeling with polyclonal antibodies raised in rabbit and guinea pig (Fig. 2E). In summary, these findings indicate that the two splice products of the Munc13-2 gene, bMunc13-2 and ubMunc13-2, are targeted to different types of synapses. More precisely, ubMunc13-2 appears to be selectively localized to ribbon synapses, while bMunc13-2 is present at conventional chemical synapses that are likely formed by amacrine cells.

\section{Imaging fluorescently tagged Munc13 variants in knock-in mice validates immunostaining data}

We next turned to knock-in mice expressing C-terminally EXFPtagged Munc13-1 (Munc13-1-EYFP) (Kalla et al., 2006), Munc13-2, and Munc13-3 to investigate the distribution pattern of Munc13 isoforms independently of the use of our isoformspecific antibodies. Munc13-2-EYFP and Munc13-3-EGFP lines were engineered by replacing the endogenous STOP codon in the last coding exon of the respective genes with an EYFP/EGFPSTOP cassette in-frame (see Materials and Methods) (Fig. 4A$F)$. Since the EYFP coding sequence was added right after the Munc13-2 coding sequence, both ubMunc13-2 and bMunc13-2 splice variants are tagged with EYFP in the Munc13-2-EYFP line.

As the intrinsic fluorescent signal in the knock-in mice was rather weak, immunostaining of the tag was performed. In all cases, fluorescence distribution in the knock-in mice was comparable with that observed by immunolabeling in wild-type mice, with the exception of artifactual blood vessel labeling that is at-

of a differential synaptic localization. $\boldsymbol{B}, \boldsymbol{C}$, ubMunc13-2 distribution extensively overlapped with that of the ribbon protein $\mathrm{CHBP} 2$ and of the vesicular glutamate transporter VGluT1, revealing its presence at ribbon synapses of the OPL $(\boldsymbol{B})$ and the IPL (C). D, No such association was observed with bMunc13-2. E, A polyclonal antibody raised in guinea pig against bMunc13-2 yielded strictly the same labeling pattern as the rabbit polyclonal antibody used otherwise (left). Upon double labeling for bMunc13-2 and ubMunc13-2, no overlap was observed (middle and right panels). ONL, INL, Outer and inner nuclear layers; OPL, IPL, outer and inner plexiform layers; $G C L$, ganglion cell layer. Scale bars: $A, E, 10 \mu \mathrm{m} ; \boldsymbol{B}-\boldsymbol{D}, 2 \mu \mathrm{m}$. 


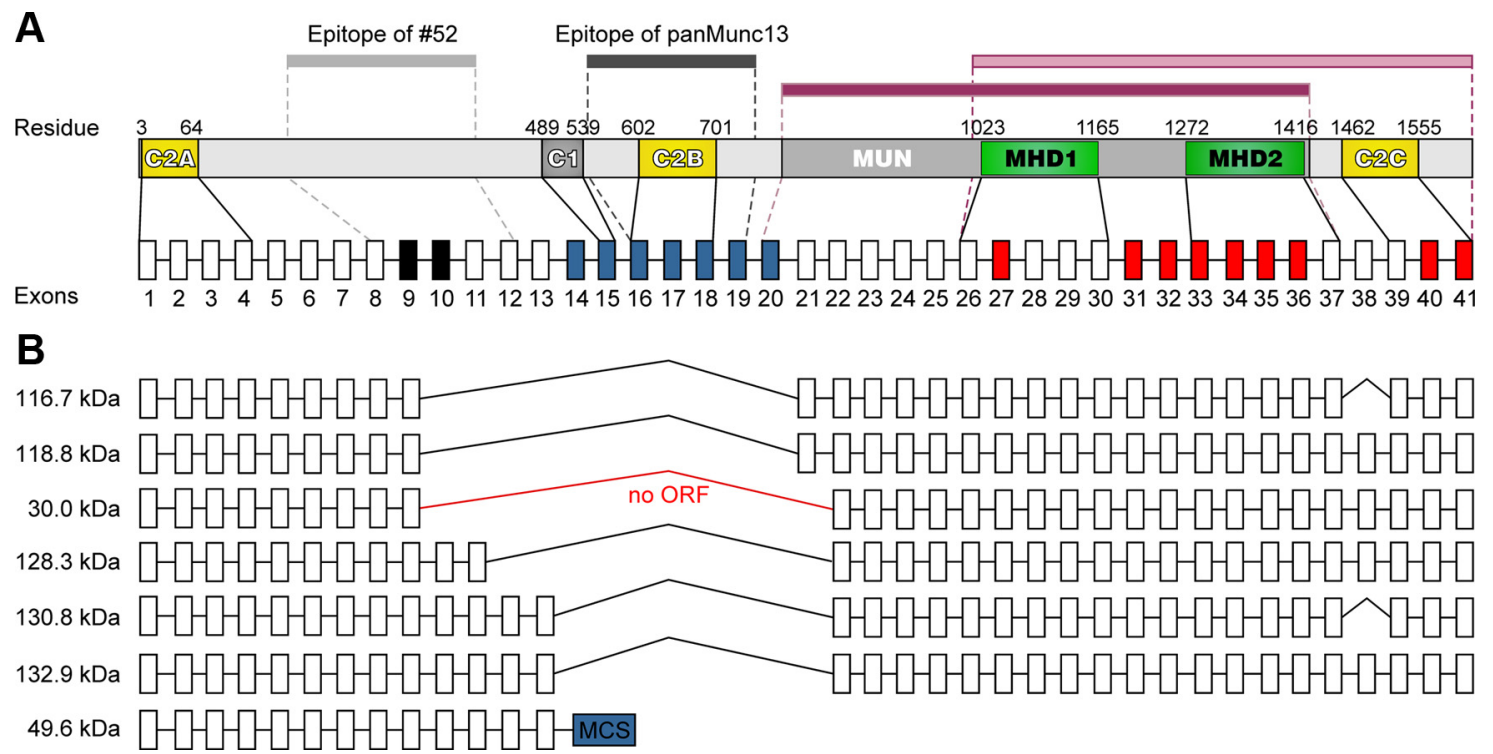

Figure 3. Analysis of ubMunc13-2 transcripts in Munc13-2 KO retina. A, Domain structure of mouse ubMunc13-2 with relevant amino acid residue numbers. The bars above the domain structure represent the antigens used to raise the antibodies used in the present study [52, light gray, residues 182-408; panMunc13 TL (BD Biosciences), dark gray, residues 544-757], the MUN domain required for priming function in neurons as defined by Basu et al. (2005) (light pink, residues 782-1421), and the minimal Munc13 priming domain required in chromaffin cells as defined by Stevens et al. (2005) (dark pink, residues 1017-1601). The exons (not drawn to scale) encoding ubMunc13-2 are shown below the domain structure. The stippled and solid lines indicate the exons that encode the respective ubMunc13-2 sequence stretch. The black and red coloring indicates exons in which forward and reverse primers for the PCR analysis of Munc13-2 K0 retina CDNA were located (Table 1). The blue coloring indicates exons that are deleted by the Munc13-2 K0 strategy. C1, C1 domain; C2, C2 domain; MHD, Munc13 homology domain (Koch et al., 2000); MUN, Mun domain (Li et al., 2011). B, ubMunc13-2 transcripts found in Munc13-2 KO retina. Predicted molecular weights of the corresponding protein products are indicated on the left. Note that the sequences $5{ }^{\prime}$ to exon 9 were not PCR amplified and have been added to the image, assuming that the corresponding transcripts were generated from the standard and only known transcriptional start site. MCS, Multicloning site of the Munc13-2 KO targeting vector; ORF, open reading frame.

tributable to the EYFP antibody used (Fig. $4 H$, asterisks). In the OPL, only Munc13-2-EYFP, but not Munc13-1-EYFP or Munc13-3-EGFP, could be detected; upon combined immunostaining with the anti-Munc13 antibodies, the Munc13-2-EYFP signal essentially overlapped with ubMunc13-2 (Fig. 4I, top), confirming that ubMunc13-2 is solely expressed at photoreceptor ribbon synapses. In the IPL, Munc13-2-EYFP immunoreactivity overlapped with that of ubMunc13-2 (Fig. $4 I$, bottom) and of bMunc13-2 (data not shown). Together, these data corroborate the exclusive expression of ubMunc13-2 at ribbon synapses in the OPL.

\section{ubMunc13-2 is located at the active zone of the photoreceptor ribbon synapse}

High-resolution imaging using STED microscopy confirmed that ubMunc13-2 labeling is closely apposed to, but does not overlap with the ribbon marker $\mathrm{CtBP} 2$, indicating that the protein may localize along the base of the ribbon and not to the ribbon itself (Fig. 5A). Indeed, at the ultrastructural level, ubMunc13-2 was found to be located at the presynaptic membrane forming the membrane invagination at the ribbon site and frequently at the rim of the CAZ-classically regarded as the main fusion site for SVs (Fig. 5B,C,E; $n=150$ photoreceptor synapses; total number of gold particles, 139). Occasionally membrane-associated ubMunc13-2 was observed at distances of up to $250 \mathrm{~nm}$ from the CAZ (data not shown). Of note, ubMunc13-2 labeling decorated both rods and cones terminals (Fig. $5 B$, insets), in agreement with our observations at the lightmicroscopy level (Fig. 2 B).

Lack of ubMunc13-2 has little effect on photoreceptor ribbon synaptic transmission

The exclusive presence of ubMunc13-2 at the active zone of photoreceptor ribbon synapses and the absence of other Munc13s from these structures implies that ubMunc13-2 may have a crucial role in regulating synaptic release, as reported for its closest homolog Munc13-1 at hippocampal synapses (Augustin et al., 1999b). To investigate this possibility, retinal activity was monitored by performing ERG recordings under scotopic and photopic conditions in wild-type and Munc13-2 KO littermates, in which both bMunc13-2 and ubMunc13-2 function is abolished (Fig. 6A,B). The a-wave component of the ERG response was unaltered in Munc13-2 KO mice compared with wild-type controls, which reflects the functional integrity of the light transduction process at the photoreceptor outer segments in the Munc13-2 KO photoreceptor cells (Fig. 6A). In contrast, the b-wave, which correlates with the photoreceptor-to-ON bipolar cell synaptic transmission in the OPL (Nusinowitz et al., 2006), was slightly but significantly reduced in the KO mouse (Fig. 6A) in scotopic conditions (Fig. 6B). This correlates well with the presence of ubMunc13-2 at photoreceptor, especially rod terminals. However, b-wave implicit times were unchanged under scotopic conditions (data not shown). Under photopic conditions, the difference in b-wave amplitudes became increasingly smaller with increasing background illumination (Fig. 6B).

The ERG recordings in the Munc13-2 KO retina also revealed a slight reduction in the peak-to-peak amplitudes and frequency of oscillatory potentials, which mainly report amacrine cell activity in the IPL (data not shown). These alterations might be a consequence of the altered transmission in the OPL but could be also attributed to the absence of bMunc13-2 and ubMunc13-2 in the IPL, since both are expressed there (Figs. 2, 4). Further investigations will be necessary to dissect their respective contribution to this ERG phenotype.

We then compared the pattern of synaptic connectivity at the OPL of Munc13-2-deficient retinas and wild-type controls (Fig. $6 C-E)$. First, a triple labeling for VGluT1, PKC $\alpha$, and calbindin 


\section{A Munc13-2EYFP}

Wild-Type Gene

Targeting Vector

Mutated Gene $\left(m_{n}\right)$

Cre-Recombined Mutated Gene (m)

B

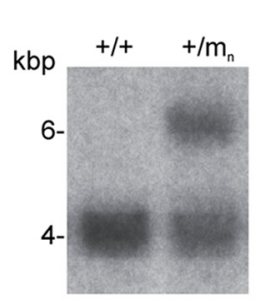

C

$170-$
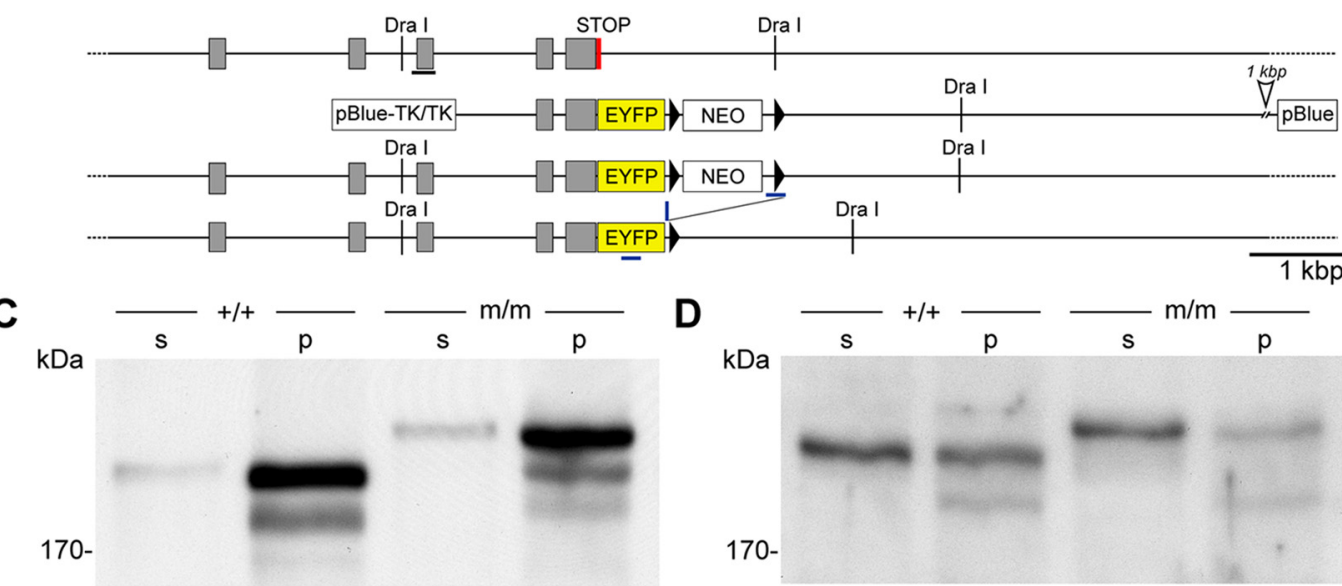

D

$\mathrm{kDa}$

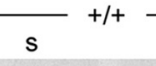

p s

$\mathrm{m} / \mathrm{m}$

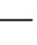

170-

\section{E Munc13-3EGFP}

Wild-Type Gene

Targeting Vector

Mutated Gene $\left(m_{n}\right)$

Cre-Recombined Mutated Gene (m)

F

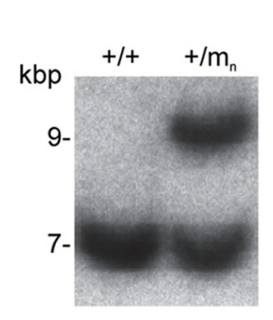

G

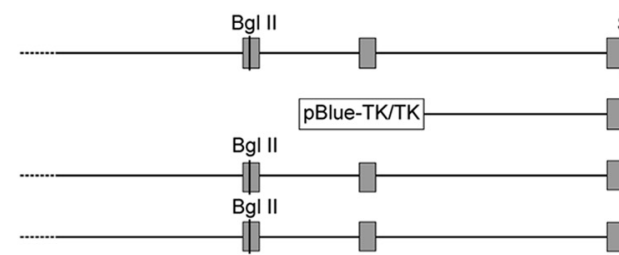

STOP

Bgl II
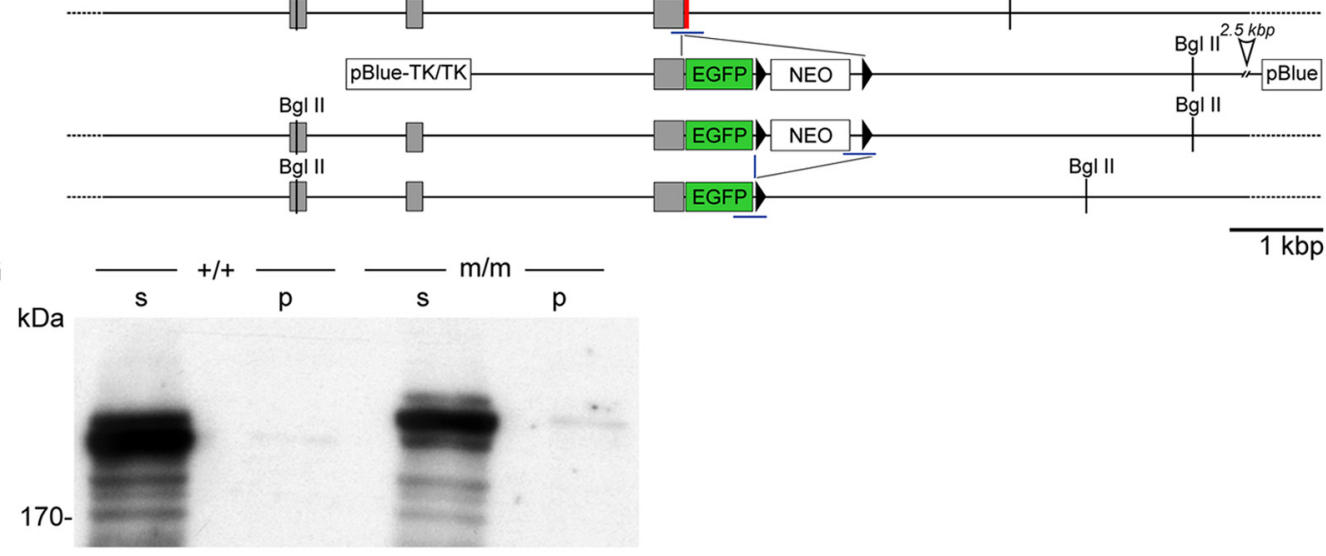

H
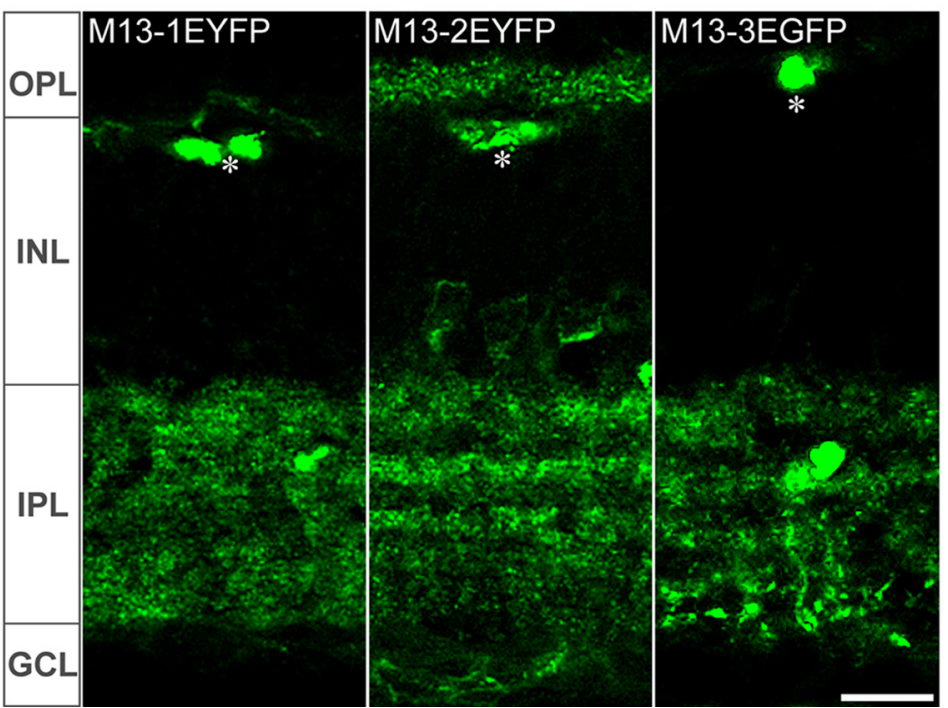

I

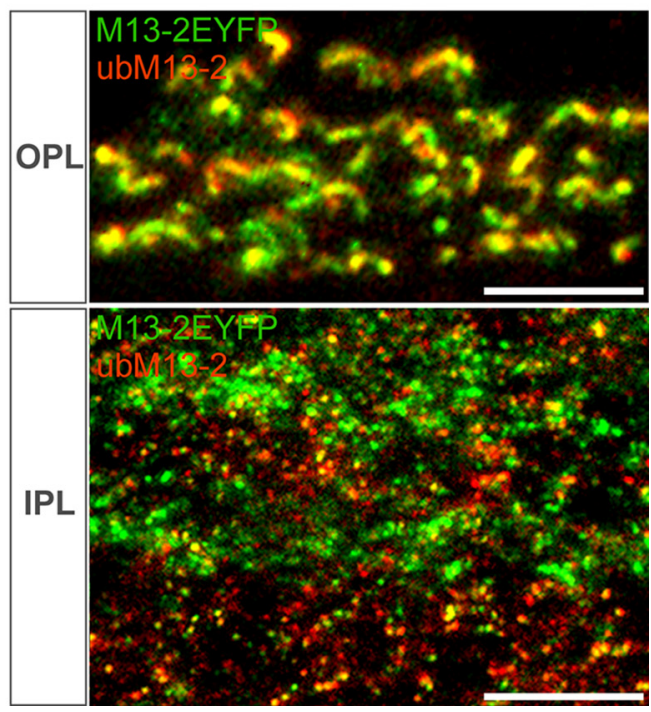

Figure 4. Generation of Munc13-2-EYFP and Munc13-3-EGFP knock-in mice. $A, E$, Strategy for the generation of the Munc13-2-EYFP $(\boldsymbol{A})$ and Munc13-3-EGFP ( $\boldsymbol{E})$ knock-in mutations in mouse embryonic stem cells. In each case, wild-type gene, targeting vector, mutated gene after homologous recombination $\left(\mathrm{m}_{\mathrm{n}}\right)$, and mutated gene after (re recombination $(\mathrm{m})$ are schematized. The gray boxes, black triangles, and a black horizontal bar indicate exons, loxP sites, and the probe used for Southern analysis of mutated genes. Sections of genomic sequence that are not shown here are indicated by an open arrowhead. NE0, Neomycin resistance gene; pBlue, pBluescript KS; TK, herpes simplex virus thymidine kinase. $\boldsymbol{B}, \boldsymbol{F}$, Southern blot analysis of Munc13-2 $(\boldsymbol{B})$ or Munc 13-3 $(\boldsymbol{F})$ wild-type and mutated genes using Dral- or Bglll-digested mouse tail DNA and the probes indicated in $A$ and $E$. C, $\boldsymbol{D}, \mathbf{G}$, Western blot analysis of brain homogenates from wild-type mice $(+/+)$ and mice carrying the mutated genes after $($ re recombination $(\mathrm{m} / \mathrm{m})$. Brains were homogenized by Ultra-Turrax, and soluble $(\mathrm{s})$ and insoluble $(\mathrm{p})$ protein fractions were separated by ultracentrifugation $(436,000 \times g)$. Proteins $(10 \mu \mathrm{g}$ per lane) were analyzed by SDS-PAGE and immunoblotting with antibodies to ubMunc13-2 (C), bMunc13-2 (D), or Munc13-3 (G). Note that Munc13-2 and Munc13-2-EYFP ( $)$ and Munc13-3 and Munc13-3-EGFP (G) protein levels were almost identical in either mouse strain. $\boldsymbol{H}, \boldsymbol{I}$, Maximum projection Z-stacks of images acquired with an Apotome microscope. $\boldsymbol{H}$, The distribution patterns of Munc13-EYFP fusion proteins correlates well with these obtained upon immunolabeling (Fig. 2). I, Colabeling for EYFP and ubMunc13-2 in Munc13-2-EYFP mice overlapped extensively at the OPL (top) but only partially at the IPL (bottom). Scale bars: $\boldsymbol{H}, 10 \mu \mathrm{m} ; \boldsymbol{I}, 5 \mu \mathrm{m}$. 

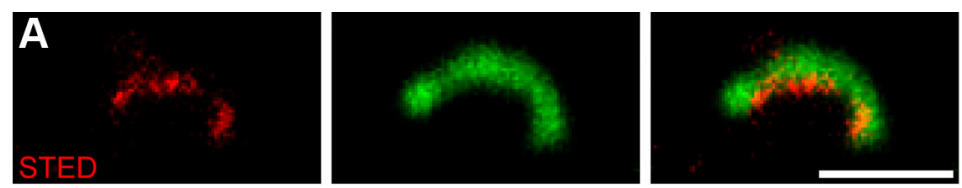

B
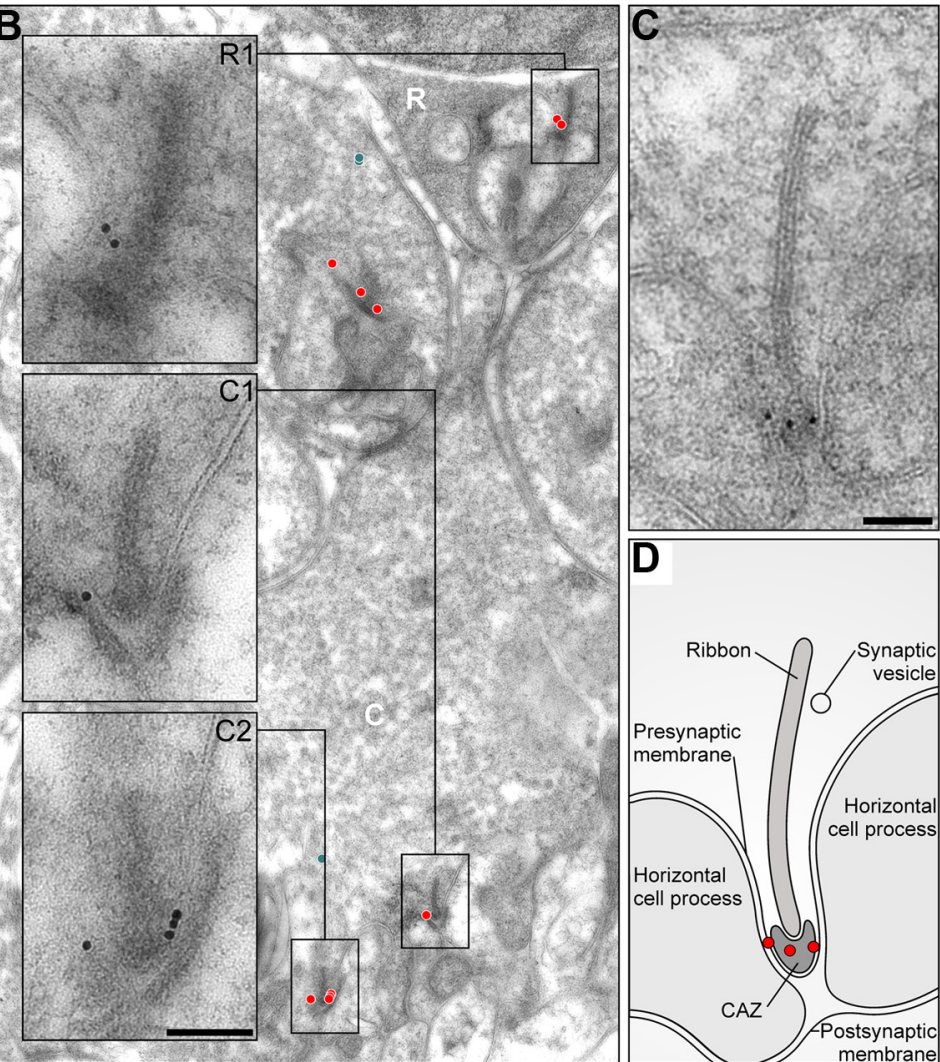

E
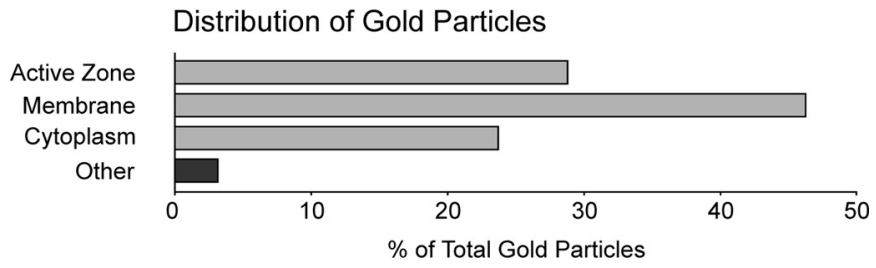

Figure 5. ubMunc13-2 localization at photoreceptor ribbon synapses. The subcellular distribution of ubMunc13-2 immunoreactivity was analyzed by STED/confocal microscopy (single-scan, in $\boldsymbol{A}$ ) and postembedding immunoelectron microscopy $(\boldsymbol{B}, \boldsymbol{C})$. $\boldsymbol{A}$, High-resolution fluorescence microscopy shows that ubMunc13-2 (in red) is distributed along the base of a photoreceptor ribbon, labeled by CtBP2 (in green), likely at the CAZ. B, At the ultrastructural level, gold particles (red circles indicate ribbon active zone-associated gold particles; blue circles indicate cytoplasmic, non-ribbon-associated gold particles) confirm the subcellular location of ubMunc13-2 at the CAZ and at the presynaptic membrane, within $250 \mathrm{~nm}$ of the (AZ at both rod (inset; R1) and cone (insets; $(1, C 2)$ photoreceptor ribbons. C, Relevant features visible on this electron micrograph are illustrated in $\boldsymbol{D}$. $\boldsymbol{E}$, Distribution (percentage) of gold particles in the various subregions of the rod photoreceptor presynaptic compartment (number of gold particles, 139 observed at a total of 150 rod synapses). Scale bars: STED, $1 \mu \mathrm{m}$; EM, $100 \mathrm{~nm}$.

[markers of photoreceptor terminals (Dick et al., 2003), rod bipolar dendrites (Greferath et al., 1990), and horizontal cells (Peichl and González-Soriano, 1994), respectively] shows that prototypical photoreceptor triads are formed and distributed normally also in the absence of Munc13-2; no ectopic photoreceptor synapses could be observed in the absence of Munc13-2 (Fig. 6C). Second, a triple labeling for the active zone marker bassoon (tom Dieck et al., 2005), the metabotropic glutamate receptor mGluR6 (Nakajima et al., 1993), and PKC $\alpha$ illustrate that both presynaptic components of the CAZ and postsynaptic receptors at bipolar dendrites are properly localized in Munc13-2 $\mathrm{KO}$ retinas (Fig. 6D). It also implies that the Munc13-2 KO b-wave phenotype is not a consequence of mGluR6 mislocaliza- tion at bipolar dendrites (Koyasu et al., 2008). Third, a triple labeling for ubMunc13-2, the ribbon-marker CtBP2, and the L-type voltage-gated calcium channel Cav1.4 illustrates that calcium channels are properly localized to the base of synaptic ribbons in the absence of ubMunc13-2 (Fig. 6E). Hence, the Munc13-2 KO b-wave phenotype is not a result of calcium channel mislocalization. Together, the normal arrangements observed at the OPL reflect the proper establishment and maintenance of the local neuronal circuitry in the absence of Munc13-2. The aforementioned impairment of photoreceptor ribbon synaptic transmission is therefore unlikely a result of aberrant synaptic connectivity.

\section{The photoreceptor ribbon synapse of Munc13-2 KOs harbors docked synaptic vesicles}

Recent studies using high-pressure-frozen, unfixed material have uncovered a loss of SVs docked at the active zone of synapses lacking members of the Unc13/Munc13 protein family in Caenorhabditis elegans unc-13 mutants (Gracheva et al., 2006; Weimer et al., 2006; Hammarlund et al., 2007) and in hippocampal neurons from Munc13-1/2 double KO mice (Siksou et al., 2009). To test whether the physiological deficit at photoreceptor ribbon synapses in the Munc13-2 KO retina is paralleled by a morphological defect at the photoreceptor synapse, we compared the ultrastructure of wild-type and Munc13-2 KO retinas processed following a comparable highpressure-freezing procedure (Möbius et al., 2010). The ultrastructural preservation of retinal tissue in the OPL (Fig. 7B) obtained by this method is superior to that obtained by classical methods using chemical fixation and room temperature dehydration for the preparation of retinal tissue for electronmicroscopic analysis (Möbius et al., 2010). We therefore propose that this method is well suited for in-depth morphological investigation of photoreceptor ribbon synaptic sites.

Upon tomographic reconstruction, the organization of photoreceptor ribbon synapses was not detectably altered in the Munc13-2 KO retina (Fig. 7). The populations of SVs docked at the plasma membrane forming the presynaptic invagination (rendered in red), tethered to the ribbon (rendered in orange) or to its base (rendered in white) were comparable (Fig. 7A-C). Both rod and cone ribbon synapses were tomographically sampled; however, SV docking was exclusively quantified in rods (Fig. $7 D$ ) to facilitate the collection of tomographic volumes for statistical analyses, since rod terminals largely outnumber cone terminals. The region analyzed at ribbon synaptic sites (15 wild-type and 14 Munc13-2 KO synapses) encompasses the CAZ, the ribbon in its entire height, and the presynaptic membrane forming the entire membrane invagina- 
A

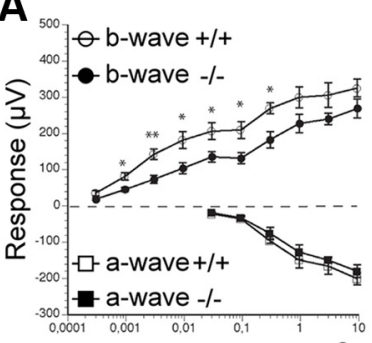

Flash Intensity $\left(\mathrm{Cds} / \mathrm{m}^{2}\right)$

C
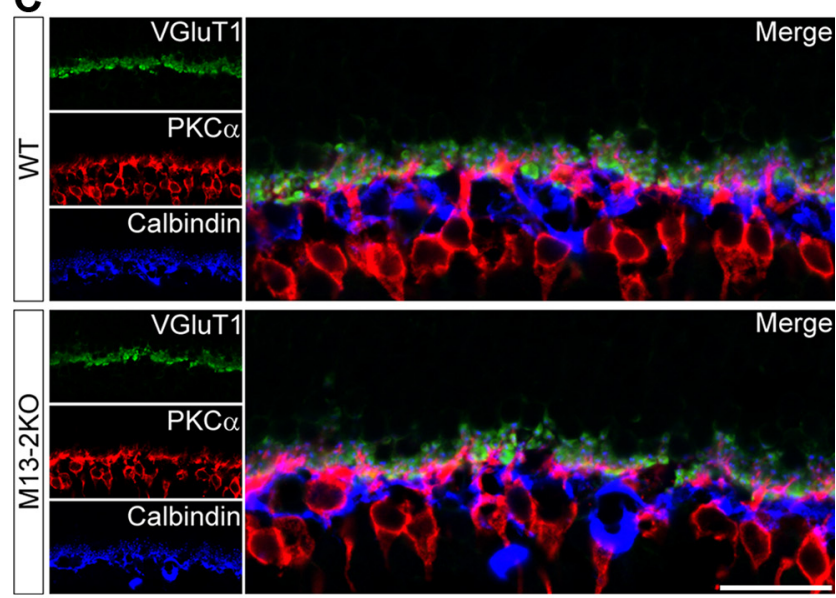

D
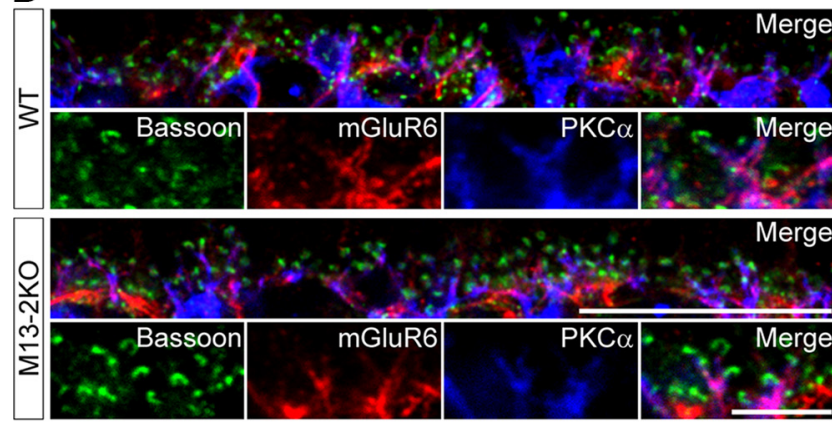

E
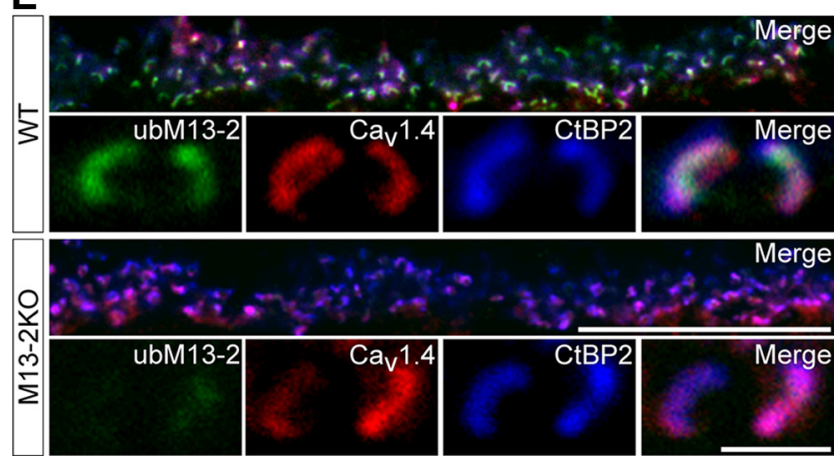

Figure 6. Deletion of Munc13-2 is detrimental to proper synaptic transmission but not to retinal organization. ERG recordings were performed in scotopic and photopic conditions. $A$, Intensity-response curves did not differ statistically for the a-waves (squares) of wild-type and Munc13-2 KO mice. In contrast, the b-wave (circles) amplitudes were slightly, but significantly reduced in the Munc13-2 KO mice compared with those of wild-type controls (mean \pm SEM; $n=5 ;{ }^{*} 0.05 \geq p>0.01 ;{ }^{* *} 0.01 \geq p>0.001$; one-way ANOVA), under scotopic (darkadapted) conditions. $\boldsymbol{B}$, Under photopic conditions, differences in b-wave amplitudes in response to high flash intensities became increasingly smaller upon increasing background illumination (open symbols, wild type; filled symbols, Munc13-2 K0). C-E, High-resolution, single-plane confocal images. C, Prototypical photoreceptor triads comprising photoreceptor terminals (labeled with VGluT1), bipolar dendrites (labeled with PKC $\alpha$ ), and horizontal cell tion (mean distance from the CAZ: WT, $324 \pm 24 \mathrm{~nm}$; Munc13-2 $\mathrm{KO}, 304 \pm 21 \mathrm{~nm}$; mean $\pm \mathrm{SEM})$. The selected regions of interest therefore correlate well with the subcellular distribution of ubMunc13-2 (Fig. 5). No significant difference was detected in either the average number of docked vesicles per $100 \mathrm{~nm}$ length of the plasma membrane (Fig. $7 A$, red vesicles; WT, $2.57 \pm 0.35$; Munc13-2 KO, 2.46 \pm 0.23 ; mean \pm SEM; $p=0.67)$, the number of docked vesicles at the base of the ribbon (Fig. 7A, white vesicles; WT, $7.23 \pm 0.32$; Munc13-2 KO, $7.15 \pm 0.32 ; p=0.86)$, or the number of ribbon-associated vesicles per $100 \mathrm{~nm}$ ribbon height (Fig. 7A, orange vesicles; WT, $15.67 \pm 1.01$; Munc13-2 KO, $15.89 \pm 1.55$; mean $\pm \mathrm{SEM} ; p=0.86)$. These observations parallel the relatively mild phenotypic change observed by ERG recordings and indicate that synaptic release is likely only very mildly affected in photoreceptor ribbon synapses in the absence of ubMunc13-2, or indeed in the absence of any priming factor of the Munc13 family.

\section{Discussion}

Munc13s are SV priming proteins at presynaptic active zones (Südhof and Rizo, 2011). Several studies characterized Munc13 function by overexpressing or deleting them in heterologous cells or cultured neurons, but little is known about Munc13 localization and function in intact neuronal networks (Song et al., 1998; Augustin et al., 2001; Varoqueaux et al., 2002, 2005; Feldmann et al., 2003; PivotPajot et al., 2008; Zhu et al., 2008). We show here that the canonical Munc13 isoforms Munc13-1, bMunc13-2, ubMunc13-2, and Munc13-3 are selectively targeted to different types of synapses in the mouse retina (Fig. 2), and that the two Munc13-2 splice variants, bMunc13-2 and ubMunc13-2, are targeted to different types of retinal synapses, with ubMunc13-2 being specifically localized at photoreceptors and bipolar cell ribbon synapses (Fig. 2). Indeed, ubMunc13-2 is the only Munc13 present at photoreceptor ribbon synapses. Unexpectedly, however, ubMunc13-2 loss from these synapses does not abolish transmitter release according to ERG recordings (Fig. 6), indicating that SV priming at photoreceptor ribbon synapses operates partly in a Munc13-independent manner.

Along with bMunc13-2, ubMunc13-2 is one of the two major Munc13-2 splice variants (Brose et al., 1995; Song et al., 1998; Augustin et al., 1999a; Betz et al., 2001). Initially detected in human renal mesangial and cortical epithelial cells (Song et al., 1998), it is also present in mouse neuromuscular junctions (Varoqueaux et al., 2005) and brain (Betz et al., 2001; AndrewsZwilling et al., 2006; Breustedt et al., 2010), and at low levels in human neutrophils (Pivot-Pajot et al., 2008), bovine chromaffin cells (Zikich et al., 2008), and mouse airway goblet cells (Zhu et al., 2008). Using purified, isoform-specific antibodies (Figs. 1, 2), and the Munc13-1-EYFP (Kalla et al., 2006), Munc13-2EYFP, and Munc13-3-EGFP knock-in mice (Fig. 4), we show that ubMunc13-2 is the only Munc13 isoform specifically present at ribbon synapses of photoreceptors (Fig. 2). Two studies (tom Dieck et al., 2005; Limbach et al., 2011) reported Munc13-1 expression at photoreceptor ribbon synapses (but see Schmitz et al., 2001). An explanation for these findings is that the antibodies used there detected both Munc13-1 and ubMunc13-2, since

$\leftarrow$

processes (labeled with calbindin) were observed with the expected lamination at the OPL of wild-type and Munc13-2 K0 retinas. D, In PKC $\alpha$-labeled bipolar dendrites, metabotropic glutamate receptors (labeled with mGluR6) are concentrated in apposition to ribbon-bearing presynaptic active zones (labeled with bassoon) in wild-type and Munc13-2 K0 retinas. $\boldsymbol{E}$, L-Type voltage-gated calcium channels Cav1.4 are distributed at the base of CtBP2-immunoreactive photoreceptor ribbons in wild-type and Munc13-2 K0 mice. Scale bar, $20 \mu \mathrm{m}$. 

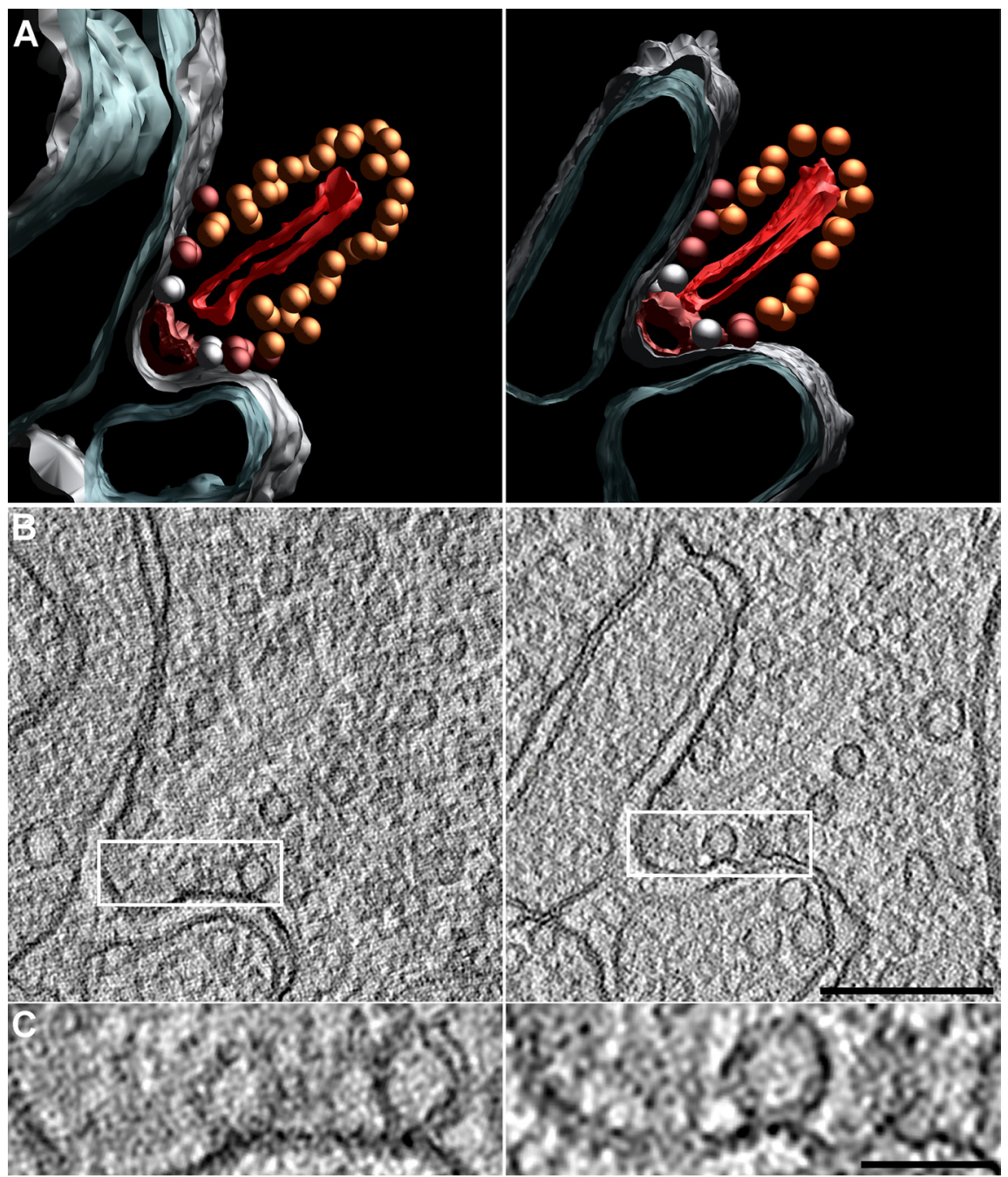

D
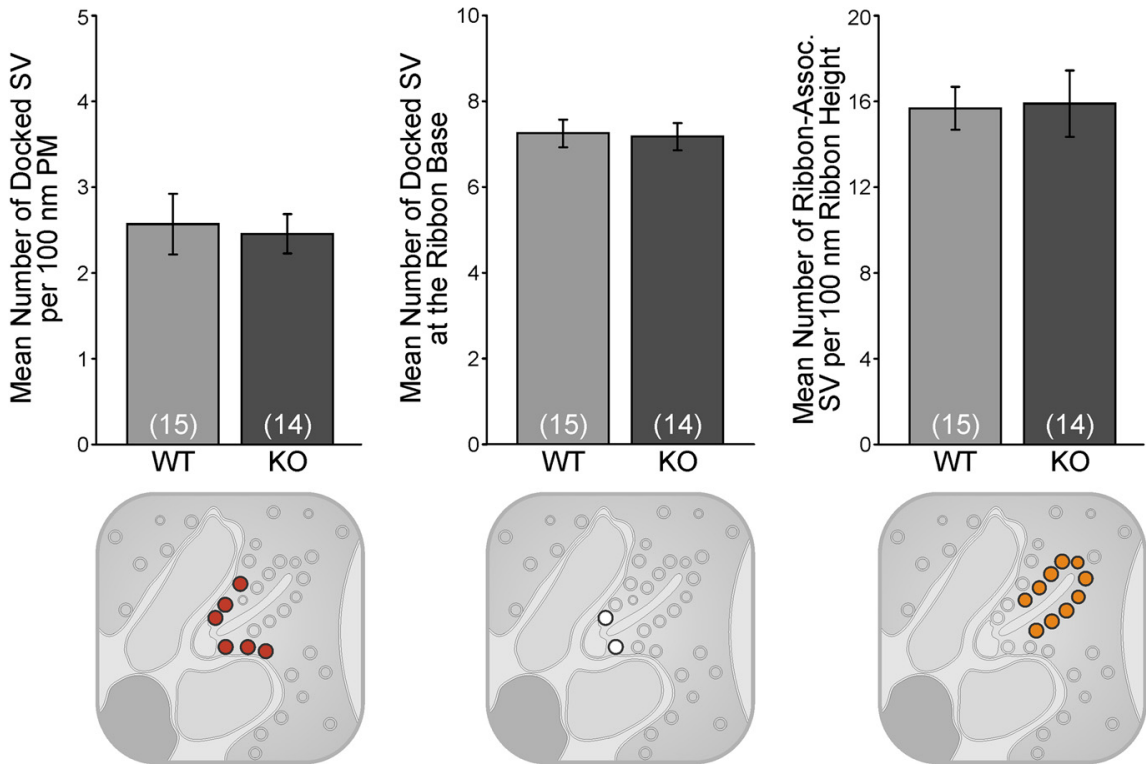

Figure 7. Tomographic reconstruction of wild-type and Munc13-2-deficient photoreceptor synapses. A, Color-coded model representing rod photoreceptor ribbon synapses from wild-type and Munc13-2 K0 retinas tomographically reconstructed from $150-\mathrm{nm}$-thick sections. $\boldsymbol{B}$, Single tomographic slice (isotropic voxel size, $1.3 \mathrm{~nm}$ ) of a rod photoreceptor ribbon synapse from wild-type (left) or Munc13-2 deletion mutant (right) retinas, at the level of the active zone. $\boldsymbol{C}$, Detail from $\boldsymbol{B}$ showing docked synaptic vesicles at the plasma membrane of wild-type or Munc13-2 deletion-mutant synapses. D, Quantitative analysis of they had been raised against the $\mathrm{C}$ terminus of Munc13-1 (tom Dieck et al., 2005), which is very similar to that of Munc13-2 and Munc13-3 (Fig. $1 B, C$, illustrating the crossreactivity of the polyclonal anti-Munc13 antibody with Munc13-1, Munc13-2, and Munc13-3), or against the $\mathrm{N}$ terminus of Munc13-1 (Limbach et al., 2011), which is highly homologous to ubMunc13-2. Indeed, the latter study acknowledges significant cross-reactivity of the antibody used with ubMunc13-2 (Limbach et al., 2011).

High-resolution imaging at the lightand electron-microscopic levels showed that ubMunc13-2 is not directly associated with ribbons, but discretely localized at the CAZ (Limbach et al., 2011) and along the plasma membrane of the invagination (Fig. 5). Immunogold labeling was observed at the presynaptic membrane up to $250 \mathrm{~nm}$ away from the CAZ, albeit at a reduced frequency (data not shown). As the SVs docked to the presynaptic membrane at the base of the ribbon, closest to the sites of calcium entry through L-type calcium channels, are thought to be the first released in response to a depolarizing stimulus (von Gersdorff, 2001), our findings are consistent with a putative role of ubMunc13-2 in SV docking or priming at this synapse.

Interestingly, morphological analyses failed to detect a difference in local OPL connectivity (Fig. 6C-E) or SV docking (Fig. 7) in Munc13-2 KO retinas. Analyses of SV docking were performed at subcellular sites where ubMunc13-2 is localized, and at sites where vesicle fusion takes place. As SVs located higher up along the ribbon may also contribute to the slower kinetic component of release (von Gersdorff, 2001), we defined and scrutinized different populations of SVs as either (1) docked at the base of the ribbon, (2) docked at the membrane forming the presynaptic invagination, or (3) ribbon-associated but not in membrane contact. The region of interest chosen for analyzing vesicle docking/ tethering largely encompasses the known subcellular distribution of ubMunc13-2 within the photoreceptor terminal and correlates closely with previously described di-

\section{$\leftarrow$}

synaptic vesicle docking. The numbers of vesicles docked at the plasma membrane (PM) forming the presynaptic invagination (rendered in red; $\boldsymbol{A}$ ), tethered to the ribbon (rendered in orange; $\boldsymbol{A}$ ) or to its base (rendered in white; $\boldsymbol{A}$ ) were comparable in wild-type ( $n=15$ synapses/tomograms) or Munc13-2 K0 ( $n=14$ synapses/tomograms) synapses. For clarity, small sketches representing the different populations of vesicles scrutinized are added below the respective graphs. Error bars indicate SEM. Scale bars: $\boldsymbol{B}, 200 \mathrm{~nm} ; \boldsymbol{C}, 50 \mathrm{~nm}$. 
mensions of ribbon-associated active zones (Midorikawa et al., 2007; Jackman et al., 2009). However, no docking deficit or reduction in the number of ribbon-associated vesicles was observed in the Munc13-2 KO.

At ribbon synapses from bipolar and hair cells, vesicles located at ribbon-free "hot spots" may also contribute to release (Midorikawa et al., 2007; Jackman et al., 2009), but we did not detect ubMunc13-2 at specific release sites remote from the canonical active zone. We cannot exclude that ubMunc13-2 is present at levels too low to be detected by immunogold labeling at such sites, and that extra-ribbon docking sites might exist at photoreceptor terminals. Yet, even if they existed and were regulated by ubMunc13-2, they would be predicted to have negligible impact on total transmission.

Using the ubMunc13-2 antibody in Munc13-2 KO retina, we detected a synaptically targeted $72 \mathrm{kDa}$ protein at $3.6 \%$ of the wild-type ubMunc13-2 level (Fig. 1D,E). This fragment likely arises from partial proteolytic degradation of a precursor generated by one of the aberrant transcripts that we identified in Munc13-2 KO retina (Fig. 3), and is most likely nonfunctional. It is predicted to contain the $\mathrm{N}$-terminal $\mathrm{C} 2 \mathrm{~A}$ domain of ubMunc13-2 and flanking sequences, which are required for synaptic targeting (Andrews-Zwilling et al., 2006), and at least the residues encoded by exon 9 (amino acids 188-266) as it is detected by the antibody 52 (Fig. 3). Thus, the $72 \mathrm{kDa}$ fragment should contain the entire N-terminal ubMunc13-2 sequence until residue 266 , with a calculated molecular weight of $30.5 \mathrm{kDa}$. To be functional, it would also have to contain the minimal C-terminal sequences required for basal priming function as determined for Munc13-1 in neurons (Basu et al., 2005) and chromaffin cells (Stevens et al., 2005), with a calculated molecular weight of 73.2 or $66.7 \mathrm{kDa}$, respectively. Thus, the entire detected fragment would have to have a molecular weight of at least 97.2 $\mathrm{kDa}$ to be functional, which is considerably larger than its actual size. We therefore conclude that this fragment cannot contain a functional $\mathrm{C}$ terminus. Indeed, a similar residual fragment is expressed in Munc13-2 KO and Munc13-1/2 DKO hippocampal neurons (Fig. 1E), the latter of which exhibit a complete loss of transmitter release and SV docking (Varoqueaux et al., 2002; Siksou et al., 2009), supporting the notion that the residual ubMunc13-2 fragment has no priming activity and cannot account for the lack of a SV docking defect in Munc13-2 KO photoreceptors.

Rather, ribbon-type synapses may execute SV priming and fusion using a unique set of proteins. Transmission at photoreceptor ribbon synapses greatly differs from conventional synaptic transmission and is characterized by graded and sustained release of neurotransmitter (von Gersdorff, 2001; Parsons and Sterling, 2003). Molecular constituents of the photoreceptor ribbon synapses vary to some extent from those of conventional synapses and are likely best suited to fulfill these specific release requirements. They include the less prototypical SNAREs synaptobrevin-1 (Sherry et al., 2003) and syntaxin-3b (Morgans et al., 1996; Sherry et al., 2006; Curtis et al., 2008), and complexin-3 and -4 (Reim et al., 2009). Interestingly, the ERG phenotype reported in complexin-3/4 DKO mice (Reim et al., 2009) resembles the one observed in Munc13-2 KOs.

In contrast to a previous report, indicating that SV docking and priming reflect the same molecular process (Siksou et al., 2009), the lack of an SV docking defect along with the b-wave alteration in the Munc13-2 KO may indicate that SV docking and priming represent different molecular processes in ribbon synapses (i.e., that ubMunc13-2 has a priming role that is not related to SV docking). Indeed, ribbon-associated vesicles tethered directly to the ribbon but not in membrane contact were proposed to be primed (Heidelberger et al., 2002), representing a form of SV priming in the absence of membrane attachment. However, we neither detected ubMunc13-2 at the ribbon itself, nor any change in the ribbon-associated pool of SVs in Munc13-2 KO mice. We therefore have no evidence to indicate that ubMunc13-2 is involved in priming of these vesicles.

The localization of ubMunc13-2 at specific subsets of synapses, including retina ribbon synapses and motor endplates, is consistent with a specific role in regulating neurotransmitter release. It is possible that release at photoreceptor ribbon synapses is not as tightly regulated by ubMunc13-2, as it is regulated by Munc13-1 at most hippocampal synapses (Augustin et al., 1999b). Accordingly, synaptic transmission at the immature neuromuscular junction, where ubMunc13-2 is the most abundant Munc13 isoform, is not entirely abolished upon Munc13 deletion either (Varoqueaux et al., 2005). ubMunc13-2 may regulate the release of a subset of SVs that correlate with a specific release component, or do so only in specific physiological states that we cannot visualize further with our experimental settings. In the future, identifying and recording the kinetic release features specifically from ubMunc13-2-expressing synapses of photoreceptors, bipolar terminals, or the hippocampus will further elucidate the variety of molecular mechanisms underlying neurotransmitter release.

\section{References}

Andrews-Zwilling YS, Kawabe H, Reim K, Varoqueaux F, Brose N (2006) Binding to Rab3A-interacting molecule RIM regulates the presynaptic recruitment of Munc13-1 and ubMunc13-2. J Biol Chem 281:19720-19731.

Augustin I, Betz A, Herrmann C, Jo T, Brose N (1999a) Differential expression of two novel Munc13 proteins in rat brain. Biochem J 337:363-371.

Augustin I, Rosenmund C, Südhof TC, Brose N (1999b) Munc13-1 is essential for fusion competence of glutamatergic synaptic vesicles. Nature 400:457-461.

Augustin I, Korte S, Rickmann M, Kretzschmar HA, Südhof TC, Herms JW, Brose N (2001) The cerebellum-specific Munc13 isoform Munc13-3 regulates cerebellar synaptic transmission and motor learning in mice. J Neurosci 21:10-17.

Basu J, Shen N, Dulubova I, Lu J, Guan R, Guryev O, Grishin NV, Rosenmund C, Rizo J (2005) A minimal domain responsible for Munc13 activity. Nat Struct Mol Biol 12:1017-1018.

Betz A, Ashery U, Rickmann M, Augustin I, Neher E, Südhof TC, Rettig J, Brose N (1998) Munc13-1 is a presynaptic phorbol ester receptor that enhances neurotransmitter release. Neuron 21:123-136.

Betz A, Thakur P, Junge HJ, Ashery U, Rhee JS, Scheuss V, Rosenmund C, Rettig J, Brose N (2001) Functional interaction of the active zone proteins Munc13-1 and RIM1 in synaptic vesicle priming. Neuron 30:183-196.

Breustedt J, Gundlfinger A, Varoqueaux F, Reim K, Brose N, Schmitz D (2010) Munc13-2 differentially affects hippocampal synaptic transmission and plasticity. Cereb Cortex 20:1109-1120.

Brose N, Hofmann K, Hata Y, Südhof TC (1995) Mammalian homologues of Caenorhabditis elegans unc-13 gene define novel family of C2-domain proteins. J Biol Chem 270:25273-25280.

Curtis LB, Doneske B, Liu X, Thaller C, McNew JA, Janz R (2008) Syntaxin $3 \mathrm{~b}$ is a t-SNARE specific for ribbon synapses of the retina. J Comp Neurol 510:550-559.

Dick O, tom Dieck S, Altrock WD, Ammermüller J, Weiler R, Garner CC, Gundelfinger ED, Brandstätter JH (2003) The presynaptic active zone protein bassoon is essential for photoreceptor ribbon synapse formation in the retina. Neuron 37:775-786.

Dimova K, Kawabe H, Betz A, Brose N, Jahn O (2006) Characterization of 
the Munc13-calmodulin interaction by photoaffinity labeling. Biochim Biophys Acta 1763:1256-1265.

Feldmann J, Callebaut I, Raposo G, Certain S, Bacq D, Dumont C, Lambert N, Ouachée-Chardin M, Chedeville G, Tamary H, Minard-Colin V, Vilmer E, Blanche S, Le Deist F, Fischer A, de Saint Basile G (2003) Munc13-4 is essential for cytolytic granules fusion and is mutated in a form of familial hemophagocytic lymphohistiocytosis (FHL3). Cell 115:461-473.

Gracheva EO, Burdina AO, Holgado AM, Berthelot-Grosjean M, Ackley BD, Hadwiger G, Nonet ML, Weimer RM, Richmond JE (2006) Tomosyn inhibits synaptic vesicle priming in Caenorhabditis elegans. PLoS Biol 4:e261.

Greferath U, Grünert U, Wässle H (1990) Rod bipolar cells in the mammalian retina show protein kinase C-like immunoreactivity. J Comp Neurol 301:433-442.

Gundelfinger ED, Fejtova A (2011) Molecular organization and plasticity of the cytomatrix at the active zone. Curr Opin Neurobiol. Advance online publication. Retrieved April 24, 2012. doi:10.1016/j.conb. 2011.10.005.

Hammarlund M, Palfreyman MT, Watanabe S, Olsen S, Jorgensen EM (2007) Open syntaxin docks synaptic vesicles. PLoS Biol 5:e198.

Heidelberger R, Sterling P, Matthews G (2002) Roles of ATP in depletion and replenishment of the releasable pool of synaptic vesicles. J Neurophysiol 88:98-106.

Jackman SL, Choi SY, Thoreson WB, Rabl K, Bartoletti TM, Kramer RH (2009) Role of the synaptic ribbon in transmitting the cone light response. Nat Neurosci 12:303-310.

Johnson J, Tian N, Caywood MS, Reimer RJ, Edwards RH, Copenhagen DR (2003) Vesicular neurotransmitter transporter expression in developing postnatal rodent retina: GABA and glycine precede glutamate. J Neurosci 23:518-529.

Kalla S, Stern M, Basu J, Varoqueaux F, Reim K, Rosenmund C, Ziv NE, Brose N (2006) Molecular dynamics of a presynaptic active zone protein studied in Munc13-1-enhanced yellow fluorescent protein knock-in mutant mice. J Neurosci 26:13054-13066.

Koch H, Hofmann K, Brose N (2000) Definition of Munc13-homologydomains and characterization of a novel ubiquitously expressed Munc13 isoform. Biochem J 349:247-253.

Koyasu T, Kondo M, Miyata K, Ueno S, Miyata T, Nishizawa Y, Terasaki H (2008) Photopic electroretinograms of mGluR6-deficient mice. Curr Eye Res 33:91-99.

Kremer JR, Mastronarde DN, McIntosh JR (1996) Computer visualization of three-dimensional image data using IMOD. J Struct Biol 116:71-76.

Lakso M, Pichel JG, Gorman JR, Sauer B, Okamoto Y, Lee E, Alt FW, Westphal H (1996) Efficient in vivo manipulation of mouse genomic sequences at the zygote stage. Proc Natl Acad Sci U S A 93:58605865.

Li W, Ma C, Guan R, Xu Y, Tomchick DR, Rizo J (2011) The crystal structure of a Munc13 C-terminal module exhibits a remarkable similarity to vesicle tethering factors. Structure 19:1443-1455.

Limbach C, Laue MM, Wang X, Hu B, Thiede N, Hultqvist G, Kilimann MW (2011) Molecular in situ topology of Aczonin/Piccolo and associated proteins at the mammalian neurotransmitter release site. Proc Natl Acad Sci U S A 108:E392-E401.

Mastronarde DN (1997) Dual-axis tomography: an approach with alignment methods that preserve resolution. J Struct Biol 120:343352.

Matsubara A, Laake JH, Davanger S, Usami S, Ottersen OP (1996) Organization of AMPA receptor subunits at a glutamate synapse: a quantitative immunogold analysis of hair cell synapses in the rat organ of Corti. J Neurosci 16:4457-4467.

Matthews G, Fuchs P (2010) The diverse roles of ribbon synapses in sensory neurotransmission. Nat Rev Neurosci 11:812-822.

Midorikawa M, Tsukamoto Y, Berglund K, Ishii M, Tachibana M (2007) Different roles of ribbon-associated and ribbon-free active zones in retinal bipolar cells. Nat Neurosci 10:1268-1276.

Möbius W, Cooper B, Kaufmann WA, Imig C, Ruhwedel T, Snaidero N, Saab AS, Varoqueaux F (2010) Electron microscopy of the mouse central nervous system. Methods Cell Biol 96:475-512.

Morgans CW, Brandstätter JH, Kellerman J, Betz H, Wässle H (1996) A
SNARE complex containing syntaxin 3 is present in ribbon synapses of the retina. J Neurosci 16:6713-6721.

Nakajima Y, Iwakabe H, Akazawa C, Nawa H, Shigemoto R, Mizuno N, Nakanishi S (1993) Molecular characterization of a novel retinal metabotropic glutamate receptor mGluR6 with a high agonist selectivity for L-2-amino-4-phosphonobutyrate. J Biol Chem 268:11868-11873.

Nusinowitz S, Ridder WH 3rd, Pang JJ, Chang B, Noorwez SM, Kaushal S, Hauswirth WW, Heckenlively JR (2006) Cortical visual function in the rd12 mouse model of Leber congenital amarousis (LCA) after gene replacement therapy to restore retinal function. Vision Res 46:3926-3934.

Parsons TD, Sterling P (2003) Synaptic ribbon. Conveyor belt or safety belt? Neuron 37:379-382.

Peichl L, González-Soriano J (1994) Morphological types of horizontal cell in rodent retinae: a comparison of rat, mouse, gerbil, and guinea pig. Vis Neurosci 11:501-517.

Pivot-Pajot C, Varoqueaux F, de Saint Basile G, Bourgoin SG (2008) Munc13-4 regulates granule secretion in human neutrophils. J Immunol 180:6786-6797.

Reim K, Regus-Leidig H, Ammermüller J, El-Kordi A, Radyushkin K, Ehrenreich $\mathrm{H}$, Brandstätter JH, Brose N (2009) Aberrant function and structure of retinal ribbon synapses in the absence of complexin 3 and complexin 4. J Cell Sci 122:1352-1361.

Rhee JS, Betz A, Pyott S, Reim K, Varoqueaux F, Augustin I, Hesse D, Südhof TC, Takahashi M, Rosenmund C, Brose N (2002) Beta phorbol esterand diacylglycerol-induced augmentation of transmitter release is mediated by Munc13s and not by PKCs. Cell 108:121-133.

Rosenmund C, Sigler A, Augustin I, Reim K, Brose N, Rhee IS (2002) Differential control of vesicle priming and short-term plasticity by Munc13 isoforms. Neuron 33:411-424.

Schmitz F, Königstorfer A, Südhof TC (2000) RIBEYE, a component of synaptic ribbons: a protein's journey through evolution provides insight into synaptic ribbon function. Neuron 28:857-872.

Schmitz F, Augustin I, Brose N (2001) The synaptic vesicle priming protein Munc13-1 is absent from tonically active ribbon synapses of the rat retina. Brain Res 895:258-263.

Sherry DM, Wang MM, Frishman LJ (2003) Differential distribution of vesicle associated membrane protein isoforms in the mouse retina. Mol Vis 9:673-688.

Sherry DM, Mitchell R, Standifer KM, du Plessis B (2006) Distribution of plasma membrane-associated syntaxins 1 through 4 indicates distinct trafficking functions in the synaptic layers of the mouse retina. BMC Neurosci 7:54.

Shin OH, Lu J, Rhee JS, Tomchick DR, Pang ZP, Wojcik SM, Camacho-Perez M, Brose N, Machius M, Rizo J, Rosenmund C, Südhof TC (2010) Munc13 C2B domain is an activity-dependent $\mathrm{Ca}^{2+}$ regulator of synaptic exocytosis. Nat Struct Mol Biol 17:280-288.

Siksou L, Varoqueaux F, Pascual O, Triller A, Brose N, Marty S (2009) A common molecular basis for membrane docking and functional priming of synaptic vesicles. Eur J Neurosci 30:49-56.

Song Y, Ailenberg M, Silverman M (1998) Cloning of a novel gene in the human kidney homologous to rat munc13s: its potential role in diabetic nephropathy. Kidney Int 53:1689-1695.

Stevens DR, Wu ZX, Matti U, Junge HJ, Schirra C, Becherer U, Wojcik SM, Brose N, Rettig J (2005) Identification of the minimal protein domain required for priming activity of Munc13-1. Curr Biol 15:2243-2248.

Südhof TC, Rizo J (2011) Synaptic vesicle exocytosis. Cold Spring Harb Perspect Biol 3:pii:a005637.

tom Dieck S, Brandstätter JH (2006) Ribbon synapses of the retina. Cell Tissue Res 326:339-346.

tom Dieck S, Altrock WD, Kessels MM, Qualmann B, Regus H, Brauner D, Fejtová A, Bracko O, Gundelfinger ED, Brandstätter JH (2005) Molecular dissection of the photoreceptor ribbon synapse: physical interaction of Bassoon and RIBEYE is essential for the assembly of the ribbon complex. J Cell Biol 168:825-836.

Varoqueaux F, Sigler A, Rhee JS, Brose N, Enk C, Reim K, Rosenmund C (2002) Total arrest of spontaneous and evoked synaptic transmission but normal synaptogenesis in the absence of Munc13-mediated vesicle priming. Proc Natl Acad Sci U S A 99:9037-9042.

Varoqueaux F, Sons MS, Plomp JJ, Brose N (2005) Aberrant morphology 
and residual transmitter release at the Munc13-deficient mouse neuromuscular synapse. Mol Cell Biol 25:5973-5984.

Verhage M, Sørensen JB (2008) Vesicle docking in regulated exocytosis. Traffic 9:1414-1424.

von Gersdorff H (2001) Synaptic ribbons: versatile signal transducers. Neuron 29:7-10.

Wang X, Hu B, Zieba A, Neumann NG, Kasper-Sonnenberg M, Honsbein A, Hultqvist G, Conze T, Witt W, Limbach C, Geitmann M, Danielson H, Kolarow R, Niemann G, Lessmann V, Kilimann MW (2009) A protein interaction node at the neurotransmitter release site: domains of Aczonin/Piccolo, Bassoon, CAST, and rim converge on the N-terminal domain of Munc13-1. J Neurosci 29:12584-12596.
Weimer RM, Gracheva EO, Meyrignac O, Miller KG, Richmond JE, Bessereau JL (2006) UNC-13 and UNC-10/rim localize synaptic vesicles to specific membrane domains. J Neurosci 26:8040-8047.

Wojcik SM, Brose N (2007) Regulation of membrane fusion in synaptic excitation-secretion coupling: speed and accuracy matter. Neuron 55:11-24.

Zhu Y, Ehre C, Abdullah LH, Sheehan JK, Roy M, Evans CM, Dickey BF, Davis CW (2008) Munc13-2 ${ }^{-1-}$ baseline secretion defect reveals source of oligomeric mucins in mouse airways. J Physiol 586:1977-1992.

Zikich D, Mezer A, Varoqueaux F, Sheinin A, Junge HJ, Nachliel E, Melamed R, Brose N, Gutman M, Ashery U (2008) Vesicle priming and recruitment by ubMunc13-2 are differentially regulated by calcium and calmodulin. J Neurosci 28:1949-1960. 